\title{
Reflections on the logic of living systems
}

\author{
Pietro Paolo Rosas*1 \\ ${ }^{1}$ Independent researcher. Via Ungaretti 38; 09039 Villacidro (SU); Italy; tel: \\ $+39-3471014786$.
}

October 1, 2019

\begin{abstract}
This article presents an abstract model of living systems and their evolution. The Modern Synthesis describes the evolution of living systems but the debate on the origin and evolution of these systems is not yet finished. It starts from the assumption that a problem faced by any biological system is equivalent to search for a resolution of an algorithm. The concept of algorithm treated in theoretical computer science allows to define an abstract machine able to program automaton or Turing machines.

These mathematical definitions were then analyzed from a physical point 15 of view. This leads to a definition of an abstract living system that allows 16 to describe the behavior of these systems based on the energy levels and

17 entropy to which they operate. In conclusion this abstraction allows to 18 have a mathematical approach to living systems.
\end{abstract}

19 Keywords: Evolution Life Computation Energy

20 PACS 01.55.+b

${ }_{21} \quad \mathrm{MSC}(2010)$ 68Q01 68Q87 92D15

*pie.rosas@gmail.com

ORCID of the author: https://orcid.org/0000-0003-1478-9164 


\section{${ }_{22}$ Introduction}

${ }_{23}$ The biological systems in their generality represent physical structures which 24 over time tend to preserve their functions. They evolve continuously and are 25 able to adapt to the most diverse environments. This is in contrast to what ${ }_{26}$ is observed in nature for non-living processes, which are governed by paths to ${ }_{27}$ decreasing energy, whereas entropy increases.

28 At present there is not yet a unambiguous accepted theory that can explain the 29 origin and why of the behavior of living systems compared to non-living systems, 30 whereas the evolution of such systems is largely proved by the observations (Dar31 win 1859; Ferraguti and Castellacci 2011). Furthermore, an abstract approach 32 to biological systems is lacking, but some authors have made contributions in 33 this sense (Solomonoff 1964; Wolfram 2002).

${ }_{34}$ The Modern Synthesis represents the theory on the evolution of the most excit-

35 ing biological systems supported by experimental tests and certainly integrates a

36 large number of disciplines such as genetics, paleontology, comparative anatomy 37 etc. (Ferraguti and Castellacci 2011; Pigliucci and Muller 2010). However, there 38 are other hypotheses and theories that actually developing in this period but 39 the debate is certainly on and very lively (Gavrilets 2010). Some important 40 reflections concern the pattern of theory of punctuated equilibrium (Ayala and ${ }_{41}$ Avise 2014; Ayala and Bendall 1983; Gould and Eldredge 1972) regarding the 42 discontinuity in evolution. In addition symbiosis (Margulis 1970; Margulis et al. 43 1967) and coevolution (Ferraguti and Castellacci 2011), certainly represent the 44 characteristics of living systems that make their description more complex and 45 interesting.

46 The concept of organism understood as an evolutionary unit seems to be linearly

${ }_{47}$ describable with the concepts of genotype and phenotype, but in relation to the

48 evolutionary developmental biology this concept extends to the whole life cycle 
49 of an organism up to embrace the environment in which he lives (Lewontin 50 2002). It is also interesting to note the approach to a molecular evolution as ${ }_{51} \quad$ well as individual or population (Ferraguti and Castellacci 2011).

${ }_{52}$ Likewise, the concept of species does not seem to be definable in an absolute 53 sense, especially as regards unicellular organisms.

${ }_{54}$ An interesting statement that begins to lay the foundations of a possible ab55 straction and synthesis of evolution concerns the fact that an organism is seen as ${ }_{56}$ a passive object embedded in the environment, which generates problems, and a ${ }_{57}$ genetic machine within the organisms that find solutions through random com58 binations, with recombination and mutations (Ferraguti and Castellacci 2011; 59 Lewontin 2002). However, it is necessary to deepen the idea that the organism ${ }_{60}$ is seen as a passive object. In the present paper a different formulation of or${ }_{61}$ ganism will be presented. Furthermore, it is observed that organisms modify 62 the environment in which they live by influencing the selective pressures of suc${ }_{63}$ cessive generations (Lewontin 1983). A theory that combines these ideas and 64 generalizes them is that living systems solve problems.

${ }_{65}$ In this paper one presents an abstract, physical-mathematical description of ${ }_{66}$ these systems, regardless of the chemical and biological structures of which they 67 are composed and of their organization. In short, every living system behaves ${ }_{68}$ like a thermal machine (Halliday et al. 2011; Mencuccini and Silvestrini 1987), 69 uses energy, produces work and heat. Therefore a physical and mathematical 70 description of such systems must start from a reflection on thermodynamics and ${ }_{71}$ statistical mechanics. Therefore, classical concepts of thermodynamics such as 72 heat and work (Halliday et al. 2011; Mencuccini and Silvestrini 1987) will be 73 re-defined in a different but equivalent way. This is to highlight a fundamental 74 aspect of loss of information in the first case and information vehicle in the 75 second one.

76 Thermodynamics and statistical mechanics arise from the inductive interpreta- 
tion given by the interpretation and synthesis of the results of a numerous series of experiments and then from the probabilistic study of the states of a system.

79 The present article one proposes an abstract approach to these theories.

80 Generally the alphabet is defined as a finite set of symbols (Church 1936; ${ }_{81}$ Hopcroft et al. 2001; Turing 1937). This definition is modified in this article by 82 defining a symbol as the result of the application of a terminating algorithm. A 83 symbol is therefore not postulated but instead the algorithm or the procedure 84 necessary to obtain a symbol. For example, let a shepherd who has to count his ${ }_{85}$ flock of sheep. He should force the sheep to cross a gate, recognizing them by a ${ }_{86}$ sheep model, and link the recognition to a list of numbers. The recognition algo${ }_{87}$ rithm works and the shepherd manages to count the sheep. On the other hand, if the shepherd was, for example, short-sighted and without glasses he would have great difficulty in recognizing a sheep and counting it. In this second case, the symbols defined in a classical way do not take into account the fact that the shepherd is short-sighted, while both the sheep and its biunivocal correspondence with a number remains the same. Instead, considering the algorithm that allows the shepherd to recognize a sheep and to assign this recognition to 94 a number, gives the most correct interpretation of the real process. In the field of mathematics and theoretical computer science, most of the compu96 tational models studied are currently based on an evolution of a Turing machine 97 (Turing 1937). These models basically need a defined alphabet and a table in 98 which a program is stored and a memory or tape on which these symbols are written and read. 
culability, while this machine represents the abstraction of a classical concept of thermodynamics, a thermal machine (Halliday et al. 2011; Mencuccini and Silvestrini 1987).

All biological systems are composed of molecules formed by chemical elements and each reaction within their metabolism corresponds to a chemical reaction. Regardless of the type of molecule they contain, it is fundamental to discuss the reaction dynamics. The most interesting models to start from are those concerning the potential energy surfaces (Atkins and De Paula 2010; Chang and Miller 1990) and the dynamics of electron transfer (Marcus 1956). On the basis of these models it is possible to construct a geometric generalization of the potential energy surfaces, that are usually defined as energy hypersurfaces.

Another important theoretical tool, used in this article is information theory (Hartley 1928; Shannon 1948). An important aspect of this theory is represented by a definition of entropy that starts from information rather than from the physical state of a system (Gray 2011). There is therefore a link between information theory and statistical mechanics (Jaynes 1957; Katz 1967).

A fundamental concept in physical systems and in particular in living systems concerns randomness. The definition of a random sequence of symbols is found in (Martin-Löf 1966), while for the randomness of a string, that is a set of groups of symbols, the definition is due to A. N. Kolmogorov (Li and Vitányi 2008; Zvonkin and Levin 1970). A still different approach to randomness is found in Ref. (Schnorr 1971).

Given an algorithm it is important to measure how complex its execution is from a computational point of view. To solve this problem there are two approaches, one given by the theory of computational complexity, which considers the resources in terms of time and memory that are necessary for the execution of an algorithm (Arora and Barak 2009) and another, given by the Theory of the Complexity of Solomonoff-Kolmogorov-Chaitin that instead takes into ac- 
count the minimal information associated with a algorithm (Chaitin 1975; Li and Vitányi 2008) .This theory is also called Algorithmic Information Theory, for a brief review see Ref. (Hutter 2007). Concerning the Theory of Complexity of Solomonoff-Kolmogorov-Chaitin (Li and Vitányi 2008), an interpretation of the Algorithmic Information Theory through statistical mechanics is interesting (Tadaki 2008). The complexity of an algorithm is essential for systems that seek a termination of computing as living systems do. As will be seen below, the non-termination of an algorithm implies a reduction in the survival of the living system. Regarding randomness many problems still remain unresolved (Solomonoff 2011; Zenil 2011)

There is a relationship between logical irreversibility and physical irreversibility, because the deletion of an information bit generates heat (Landauer 1961). This theoretical result was also demonstrated experimentally by Peterson et al. (Peterson et al. 2016).

In the biological field, the Modern Synthesis represents a fundamental step in the interpretation of living systems and their evolution. There is a vast literature concerning the description of living systems (Darwin 1859; Ferraguti and Castellacci 2011), but the debate is not concluded.

With regard to the origin of life, fundamental experiments have been carried out to try to demonstrate an abiotic origin of amino acids, nucleotides and cell membranes (Gould and Eldredge 1972; Hargreaves et al. 1977; Miller and Urey 1959; Parker et al. 2011). A very interesting aspect about this type of experiments concerns the fact that the chiral amonoacids that are synthesized are a racemic (Parker et al. 2014), while in biological systems this does not happen, because generally one enantiomer is observed (Bada 1985).

Finally, it is necessary to highlight the current uncertainty in the classification as living organisms of viruses and viroids, but there is also for them an evolution (Webster et al. 1992) like the one that happens for biological systems. They 
interact with the cells (Agrios 1988).

A mathematical approach to biological systems concerns the study of natural selection through the fitness of genes in which it is shown that the existing inherited variance is fundamental for reproductive success (Ferraguti and Castellacci 2011; Fisher 1958).

In summary, in this text, a new definition of what is a symbol is presented. For the mathematical part, a theoretical machine is described that can create a program, while in the physical part a special definition of energy is given and consequences are drawn on the observations. In the biological part an evolutionary hypothesis is introduced based on the hypotheses made in the mathematical and physical parts.

\section{Mathematical part. Definitions and notations}

In this part of the text the definitions necessary for the development of this article will be introduced. In order to analyze the living systems from a mathematical point of view, concepts treated in theoretical computer science will be introduced.

\section{$1.1 \quad$ Turing machine}

Formally one defines a Turing machine, hereinafter referred to as $T M$, a sextuple, (Turing 1937), $\mathcal{M}=\left\langle Q, \Gamma, b, \delta, q_{0}, F\right\rangle$, where:

Definition 1. $\quad Q$ is the finite set of control states;

$\Gamma$ is the alphabet of the symbols that can be written on the tape, besides it defines $\Sigma \subseteq \Gamma$ which is the finite set of input symbols that the Turing machine can read and write on the tape at the same time; 
Definition 2. Given an alphabet $\bar{\Gamma}$, let $k$ be the length of each word built upon $\bar{\Gamma}$. All words of lenght $k$ belong to a set indicated with $\bar{\Gamma}^{k}$. The empty string is indicated with $\varepsilon$ which is a null-length string, so one has

$$
\text { for } k=0, \quad \bar{\Gamma}^{k}=\{\varepsilon\} \text {. }
$$

Furthermore all words or strings that can be formed with $\bar{\Gamma}$ are indicated with

$$
\bar{\Gamma}^{*}=\bigcup_{k \geq 0} \bar{\Gamma}^{k}=\{\varepsilon\} \cup \bar{\Gamma}^{1} \cup \bar{\Gamma}^{2} \cup \cdots \cup \bar{\Gamma}^{k}
$$

Definition 3. Instantaneous configuration of a MT is defined a string, (Ausiello et al. 2003):

$$
c=x q y,
$$

$195 \quad$ (1) $x \in \Gamma \bar{\Gamma}^{*} \cup\{\varepsilon\}$

${ }^{1} r$ stands for right, $l$ stands for left and $S$ stands for stationary, this last character is a set, the reason is explained in Def. (8). 

2014).

$$
\lambda: Q \times \Gamma \rightarrow \mathcal{P}(Q \times \bar{\Gamma} \times D) .
$$

210

211 In this case the non-deterministic automaton, (Safra 1988), can have more than one initial state, also since the function $\lambda$ leads to the power set of $Q$. This corresponds to affirming that one can simultaneously have several possible states simultaneously, up to the whole set $Q$, including an empty state because one can have this: $\lambda \subseteq Q \times \Gamma \times Q$. This automaton has the same definitions given in (1) for the $T M$, except for the transition function $\lambda$, instead of a single value. This function is represented by a transition probability matrix, $\boldsymbol{P}(\Sigma)$, of dimension 
$k \times k$, where $k=\operatorname{card}(Q)$. This matrix, for each $\Sigma \in \Gamma$ returns a probability that the automaton, finding itself in a generic state $i$, changes to a state $j$, for $i, j \in Q$. The transition probability matrix will be:

$$
\boldsymbol{P}(\Sigma)=\left(p_{i j}(\Sigma)\right) .
$$

Given that $p(\Sigma)$ a probability, it must be:

$$
p_{i j} \geq 0,
$$

while the indices $(i, j=1, \ldots, k)$ are equal in number to the number of the possible states contained in the set $Q$. Given that for each state $i$ different states $j$ exist, each column of the matrix $\boldsymbol{P}(\Sigma)$ is precisely this condition, such a matrix must have on each column:

$$
\sum_{j=1, \ldots, k} p_{i j}(\sigma)=1 .
$$

Finally, it is necessary to introduce a vector that represents the probability that the automaton is in the state $i$, this will be given by a probability vector associated with state $i$ :

$$
\mathbf{q}_{i}=\left(p\left(i_{1}\right), \ldots, p\left(i_{k}\right)\right),
$$

furthermore each element of the vector $\mathbf{q}_{i}$, being a probability, will be:

$$
p\left(i_{k}\right) \geq 0,
$$

and the sum of the probabilities of the $i$ states in which the vector can be found must be :

$$
\sum_{i=1, \ldots, k} p\left(i_{k}\right)=1
$$




\section{On the Turing machine}

The TM defined in the previous paragraphs operates on an alphabet. In general,

all grammars operate on symbols whose set is called alphabet. For example, it is not necessary to show that the number one corresponds to a sign written on a sheet that corresponds to the abstraction of a measurable and unitary quantity, but it is universally accepted. Instead, assume that such a result is not given, but that the number one is the result of the execution of certain algorithm that returns the value one. There are infinite algorithms that will return this value, think of operations on $\mathbb{R}$.

Therefore, in general, a number can be obtained as a solution to an algorithm that uses other numbers. Instead to define an alphabet, the condition that must be postulated is that the solutions of this algorithm, that starts from the same point, must always give the same value, if performed by a computer or by a person. This example serves to show more generally that the symbol of an alphabet can be defined with these two axioms:

Definition 4. Consider any alphabet, within this alphabet one must verify that:

(1) a symbol of the alphabet is defined by executing an algorithm,

(2) for each symbol the algorithm must always return the same result.

If these two conditions occur, one can use this protocol to create an alphabet. Furthermore, the second condition implies that there is the possibility that an algorithm does not give the same result universally or does not give it at all (in the case of non-termination). If in this case there is a set that does not satisfy the second condition, not defining a symbol, it does not belong to an alphabet. For example the character $b$ defined for a TM or the 0 for natural numbers can be understood as sets of all that does not belong to the respective alphabets. 


\section{Programming machine}


PM will be explored later, however the idea is that each transition probability matrix represents the modeling of a measurement on reality.

${ }_{264}$ The $b$ character used by the TM must be redefined for a PM. The $b$ character 265 does not belong to the alphabet of a TM, but is recognized by it, so $b$ is unique 266 to TM. This reasoning does not apply to PM, since the characters of its alphabet 267 are represented in probabilistic way by Markov matrices. However, if a PM has to create a program (or table) that can be read by a TM, PM must also produce the $b$ character.

Definition 5 (Alphabet of a programming machine). It is the set of probabilities arrays of transition of the symbols of an alphabet $\Gamma$ on itself, for $\Gamma$ which belongs to the definition set of a Turing machine.

$$
A=\left\{\boldsymbol{P}_{\Gamma}\right\}=\left\{\boldsymbol{P}_{\Gamma_{1}}, \boldsymbol{P}_{\Gamma_{2}}, \ldots, \boldsymbol{P}_{\Gamma_{l}}\right\} \quad \text { with } \quad l \in \mathbb{N}
$$

273 The alphabet $A$ is recognized by a TM. So for a Turing machine one has $\Gamma \wedge \succsim \subset A$.

Example 3.1. Given the stochastic matrix:

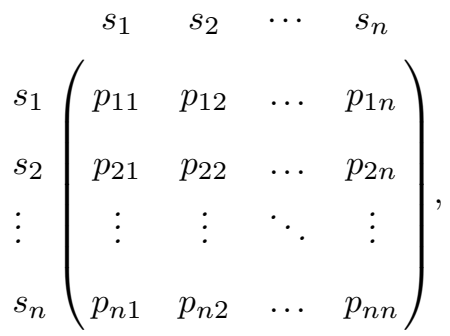

274 where $\left\{s_{1}, s_{2}, \ldots, s_{n}\right\} \in \Gamma$.

One has that, the vector ${ }^{t} \mathbf{x}_{1}=\left(p_{11}, p_{12}, \ldots, p_{1 n}\right)$ represents the probabilities of the transition of the symbol $s_{1}$ on itself and on all other symbols of the alphabet belonging to $\Gamma$. In other words, if any algorithm starts with $s_{1}$, the vector ${ }^{t} \mathbf{x}_{1}$ represents the probabilities of this algorithm ends with the symbol $s_{1}, s_{2}, \ldots, s_{n}$. Finally, one must bears in mind that each algorithm must terminate so that the symbol can be read. 
281 Thus any symbol is not considered as a priori, but represents the result of a 282 processing.

${ }_{283}$ Proposition 1. Given an alphabet $A$, read by a $P M$, it is verified that the characters 6 , read by a TM that interprets this alphabet, are all the characters that in A are represented by Markov matrices with probability distributed on all the symbols of $\Gamma$. So a PM does not have a unique symbol called $b$ in accordance with Definition (1).

Proof. Let $A$ be the alphabet of a PM, it is read and interpreted by a TM, so it must contain both the $\bar{\Gamma}$ alphabet and all the transition probability matrices of $A$. So an $A$ alphabet will consist of the symbols belonging to $\Gamma$ and a subset that does not belong to $\Gamma$. Given that a TM recognizes only an alphabet with characters almost certainly equal to themselves, one has that for a TM:

$$
\Gamma=A=\Gamma+\Gamma^{\complement},
$$

where $\Gamma^{\complement}$ represents a set of Markov matrices belonging to $A$ that do not express a symbol in a univocal way.

In accordance with Definition (1) one has:

$$
\bar{\Gamma}=\Gamma+b,
$$

as $A$ must be an alphabet recognized by a TM for the Definition (2), one writes

$$
A=\bar{\Gamma}=\Gamma+b \quad \text { and } \quad \mathrm{b}=\left(\left\{\boldsymbol{P}_{\Gamma}\right\}-\Gamma\right)=\Gamma^{\complement} .
$$

So the alphabet that it is reads by a TM corresponds to $A$ if one accepts that the set of all the semi-definite or blurred characters given by Markov matrices ${ }^{4}$ are $b$.

\footnotetext{
${ }^{4}$ For example, imagine having a disk divided into three sectors, each colored with a funda-
} 


$$
\begin{gathered}
B=\Gamma^{\complement}, \\
A=\Gamma \cup B .
\end{gathered}
$$

${ }_{294}$ Obviously for a TM that reads the set $A$ the relationship it is expected that $295 B=b$, i.e., the whole set $B$ is recognized ${ }^{5}$ by a TM only with the symbol $b$.

296 A PM therefore does not possess a b character, but rather a set of transition 297 probability matrices. If one indicates with $\boldsymbol{P}_{b} \in A$ a transient stochastic matrix, 298 so given an initial symbol a terminal symbol is not uniquely determined, then 299 one can write:

$$
B=\left\{\boldsymbol{P}_{b_{1}}, \boldsymbol{P}_{b_{2}}, \ldots, \boldsymbol{P}_{b_{n}}\right\}=\text { b } \quad \text { with } \quad n \in \mathbb{N} .
$$

Observation 3.1. If $B$ is the set of Markov matrices that are recognized as $b$ from a TM, then an empty string, composed of matrices $\boldsymbol{P}_{b}$, for a TM corresponds to:

$$
\varepsilon=\boldsymbol{P}_{b_{1}} \circ \boldsymbol{P}_{b_{2}} \circ \cdots \circ \boldsymbol{P}_{b_{n}} \quad \text { with } \quad \boldsymbol{P}_{b_{1}}, \boldsymbol{P}_{b_{2}}, \ldots, \boldsymbol{P}_{b_{n}} \in B .
$$

mental color, our eyes distinguish each of the three colors and one can create an alphabet with them. Now if the disk starts to rotate around the center the color appear there overlapped, everything appears white with different shades depending on the speed of the disc. Then a new alphabet can be formed from the three colors of the sectors plus the color given by the superposition of the three.

${ }^{5}$ The set $A$ can be read both by a TM and by a PM, but they interpret it differently. 


\subsection{States of a PM}

A PM must produce a program or an algorithm that a TM must then reproduce.

Thus also the states of the set $Q$ defined for a TM must belong also to the $Q$ states of a PM. Therefore one gets the following definition:

Definition 6. The states of a PM represent the set of transition probability matrices that has as members the $Q=\left\{q_{1}, q_{2}, \ldots, q_{n}\right\}$ states defined for a TM. So if this set is called $K$ one has:

$$
\begin{aligned}
& K=\left\{\boldsymbol{P}_{Q}\right\}=\left\{\boldsymbol{P}_{Q_{1}}, \boldsymbol{P}_{Q_{2}}, \ldots, \boldsymbol{P}_{Q_{m}}\right\} \quad \text { with } m \in \mathbb{N}, \\
& K=Q \cup \overline{\mathbf{Q}},
\end{aligned}
$$

where $\overline{\mathbf{Q}}$ is the set of Markov matrices with distributed transition probabilities on all $Q$ states, or the Markov matrices for which a state $q_{n} \in Q$ is not recognizable. From Eq. (6) one gets:

$$
\overline{\mathbf{Q}}=\left\{\boldsymbol{P}_{Q}\right\}-Q
$$

Moreover one has:

$$
q_{0} \in Q \quad \text { e } \quad F \in Q,
$$

hence the initial state and also the set of final states, as defined for a TM, belong to the set $Q$.

The set $\overline{\mathbf{Q}}$ is not defined for a $\mathrm{TM}^{6}$.

\footnotetext{
${ }^{6}$ To get an intuitive idea of the set $\overline{\mathbf{Q}}$, take this example: imagine an observer who observes the void, without having any thought on which to reason, even for a moment. This situation can be represented as $\mathbf{Q}$.
} 


\subsection{Formal definition of programming machine}

316 Taking into account the Definitions given in (1) and (4) and Equations (2) and

317 (6), one defines a programming machine:

Definition 7. Formally a $P M$ it is a 7-tuple:

$$
\mathcal{M}_{\mathcal{P}}=\left\langle K, \overline{\mathbf{Q}}, A, B, \zeta, q_{0}, F\right\rangle
$$

This machine operates on three potentially infinite ${ }^{7}$ and independent sets called state spaces:

$$
\begin{aligned}
K & =\left\{\overline{\mathbf{Q}}, q_{0}, q_{1}, \ldots, q_{n}, F\right\}, \\
A & =\left\{B, s_{1}, s_{2}, \ldots, s_{q}\right\} \\
\text { and } \quad E & =\{S, r, l\} .
\end{aligned}
$$

$K$ is the set of state transition probability matrices $Q=\left\{q_{0}, q_{1}, \ldots, q_{n}, F\right\}$ defined in Eq. (6), where the stochastic matrix is:

$$
\boldsymbol{P}_{Q}=\left(\begin{array}{cccc}
p_{11} & p_{12} & \ldots & p_{1 n} \\
p_{21} & p_{22} & \ldots & p_{2 n} \\
\vdots & \vdots & \ddots & \vdots \\
p_{n 1} & p_{n 2} & \ldots & p_{n n}
\end{array}\right)
$$

$F$ is a subset of $Q$, which represents the termination states defined in Eq.

(6) of a TM, $F=\left\{f_{1}, f_{2}, \ldots, f_{j}\right\} \subset Q$. The corresponding matrix is:

$$
\boldsymbol{P}_{F}=\left(\begin{array}{cccc}
p_{11} & p_{12} & \ldots & p_{1 j} \\
p_{21} & p_{22} & \ldots & p_{2 j} \\
\vdots & \vdots & \ddots & \vdots \\
p_{i 1} & p_{i 2} & \ldots & p_{i j}
\end{array}\right)
$$

\footnotetext{
${ }^{7}$ In this article, these sets will be considered finite.
} 


$$
\boldsymbol{P}_{\Gamma}=\left(\begin{array}{cccc}
p_{11} & p_{12} & \ldots & p_{1 r} \\
p_{21} & p_{22} & \ldots & p_{2 r} \\
\vdots & \vdots & \ddots & \vdots \\
p_{r 1} & p_{r 2} & \ldots & p_{r r}
\end{array}\right)
$$

$\zeta$ is the transition function:

$$
\zeta:(K-F) \times A \rightarrow K \times A \times E
$$

$E$ it is a finite set that contains the matrices of the transition probabilities of the one-dimensional displacements within the $n$-tuple which represents the code on which the PM operates. Let be $D^{\prime}=\{r, l\}$ then it holds ${ }^{8}$ :

$$
\begin{aligned}
& E=\left\{\boldsymbol{P}_{D^{\prime}}\right\}, \\
& \boldsymbol{P}_{D^{\prime}}={ }_{l}^{r}\left(\begin{array}{cc}
r & l \\
p_{1,1} & p_{1,2} \\
p_{2,1} & p_{2,2}
\end{array}\right),
\end{aligned}
$$

assuming that $r$ and $l$ are the shift of the head to the right and to the left, respectively, while the head remains immobile whenever the $\boldsymbol{P}_{D^{\prime}}$ matrix does not express a unique result, i.e. the stochastic matrix is not absorbing on a value of $l$ or $r$.

In this case the whole set $D$ is defined by following Definition.

\footnotetext{
${ }^{8}$ From a physical chemical point of view a so-called displacement should represent a measurable symmetry variation on hypersurfaces of energy distinguishable from that belonging to the symbol of initial measurement $\left\langle q_{0}, s\right\rangle$. Such energy hypersurfaces may have lower or higher levels of energy than the hypersurface where the variation occurred, but not equal, otherwise there would be no measurable displacement.
} 
329

A TM recognizes the elements of set $D=\{r, l, S\}$ as specified in Definition (1). One assumes for a TM, the equality $E=D$ and then one has

$$
\overline{\mathbf{D}}=S
$$

Definition 9. Keeping in mind the definition given in Eq. (1), one defines as an instantaneous configuration of a PM a string composed of matrices:

$$
\mathcal{C}=\boldsymbol{X} \circ \boldsymbol{P}_{Q} \circ \boldsymbol{Y},
$$

341 where:
342 (1) $\boldsymbol{X} \in A \circ A^{*} \cup B$;
${ }_{343}$ (2) $\boldsymbol{P}_{Q} \in K$;
${ }_{344}(3) \quad Y \in A^{*} \circ A \cup B$. 
350 Consider a probability space $(\Omega, \mathcal{F}, \mathbb{P})$

Lemma 1. Given the fifth axiom of probability theory (Kolmogorov 1956), one has

$$
p(A+B)=p(A)+p(B),
$$

351

\section{Definition 10.}

$$
\boldsymbol{T}=\left(\begin{array}{cccc}
q_{11} & q_{12} & \ldots & q_{1 m} \\
q_{21} & q_{22} & \ldots & 2 m \\
\vdots & \vdots & \ddots & \vdots \\
q_{m 1} & q_{m 2} & \ldots & q_{m m}
\end{array}\right) \text {, where } \quad q_{11}=q_{1 m}=\cdots=q_{m m}=0
$$


defined for a TM and a PM:

$$
\mathfrak{Q} \notin Q \quad \text { and } \quad \mathfrak{Q} \notin K \text {. }
$$

Then, from the direct sum

$$
\boldsymbol{U}=\boldsymbol{P}_{Q} \oplus \boldsymbol{T}
$$

one obtains a square matrix that is called matrix $U$ :

$$
\boldsymbol{U}=\left(\begin{array}{cccccccc}
p_{11} & p_{12} & \ldots & p_{1 r} & 0 & 0 & \ldots & 0 \\
p_{21} & p_{22} & \ldots & a_{2 r} & 0 & 0 & \ldots & 0 \\
\vdots & \vdots & \ddots & \vdots & 0 & 0 & \ldots & 0 \\
p_{r 1} & p_{r 2} & \ldots & p_{r r} & 0 & 0 & \ldots & 0 \\
0 & 0 & \ldots & 0 & q_{11} & q_{12} & \ldots & q_{1 m} \\
0 & 0 & \ldots & 0 & q_{21} & q_{22} & \ldots & 2 m \\
\vdots & \vdots & & \vdots & \vdots & \vdots & \ddots & \vdots \\
0 & 0 & \ldots & 0 & q_{m 1} & q_{m 2} & \ldots & q_{m m}
\end{array}\right)
$$

358 The newly defined matrix $\boldsymbol{U}$ can be divided into four blocks:

$$
U=\left(\begin{array}{c|c}
P_{Q} & L \\
\hline M & T
\end{array}\right) \quad \text { with } \quad L=\mathbf{0} \text { and } \boldsymbol{M}=\mathbf{0} .
$$

359 The blocks $\boldsymbol{P}_{Q}$ and $\boldsymbol{T}$ have already been defined respectively in Def. (8) and in

360 Eq. (10). The $\boldsymbol{L}$ and $\boldsymbol{M}$ blocks, represented by null matrices, are modified by

361 the following definition.

\section{Definition 11.}

$$
\boldsymbol{M}=\left(\begin{array}{cccc}
b_{(r+1) 1} & b_{(r+1) 2} & \ldots & b_{(r+1) r} \\
b_{(r+2) 1} & b_{(r+2) 2} & \ldots & b_{(r+2) r} \\
\vdots & \vdots & \ddots & \vdots \\
b_{(r+m) 1} & b_{(r+m) 2} & \ldots & b_{(r+m) r}
\end{array}\right)
$$


Where for each index $i \in \mathbb{N}_{r+m}$ e $j \in \mathbb{N}_{r}$ has the elements:

$$
\left(b_{i j}\right)=\{0,1\} \quad \text { and } \quad \sum_{j=1}^{r} b_{i j}=1 .
$$

362 Now let's consider the block $\boldsymbol{L}$ of the matrix $\boldsymbol{U}$. This block is replaced with a 363 matrix that contains the probabilities of transition that link the symbols to a 364 transition code.

Definition 12. The matrix is then defined as follows:

$$
\boldsymbol{L}=\left(\begin{array}{cccc}
l_{1(r+1)} & l_{1(r+2)} & \ldots & l_{1(r+m)} \\
l_{2(r+1)} & l_{2(r+2)} & \ldots & l_{2(r+m)} \\
\vdots & \vdots & \ddots & \vdots \\
l_{r(r+1)} & l_{r(r+2)} & \ldots & l_{r(r+m)}
\end{array}\right)
$$

There is the matrix $U$ is, a stochastic matrix must be for each $i \in \mathbb{N}_{r}$ and $j \in \mathbb{N}_{r+m}:$

$$
\sum_{j=1}^{r} p_{i j}+\sum_{j=r+1}^{m} l_{i j}=1 .
$$

Then substituting with Eqs. 19 and 21 in Eq. 18, the null blocks of the stochastic matrix $\boldsymbol{U}$, one obtains the matrix of states:

$$
\boldsymbol{U}=\left(\begin{array}{cccccccc}
p_{11} & p_{12} & \ldots & p_{1 r} & l_{1(r+1)} & l_{1(r+2)} & \ldots & l_{1(r+m)} \\
p_{21} & p_{22} & \ldots & p_{2 r} & l_{2(r+1)} & l_{2(r+1)} & \ldots & l_{2(r+m)} \\
\vdots & \vdots & \ddots & \vdots & \vdots & \vdots & & \vdots \\
p_{r 1} & p_{r 2} & \ldots & p_{r r} & l_{r(r+1)} & l_{r(r+2)} & \ldots & l_{r(r+m)} \\
b_{(r+1) 1} & b_{(r+1) 2} & \ldots & b_{(r+1) r} & 0 & 0 & \ldots & 0 \\
b_{(r+2) 1} & b_{(r+2) 2} & \ldots & b_{(r+2) r} & 0 & 0 & \ldots & 0 \\
\vdots & \vdots & & \vdots & \vdots & \vdots & \ddots & \vdots \\
b_{(r+m) 1} & b_{(r+m) 2} & \ldots & b_{(r+m) r} & 0 & 0 & \ldots & 0
\end{array}\right)
$$

365 The matrix $\boldsymbol{U}$ is still a stochastic matrix, moreover this matrix represents the 


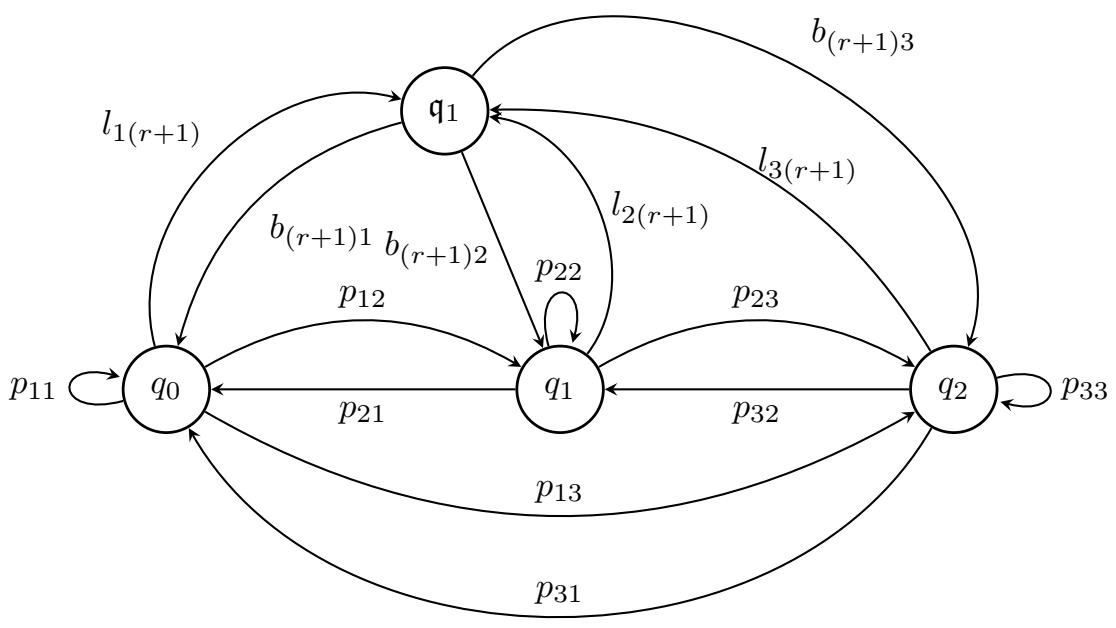

Figure 1: Digraph of a matrix $\boldsymbol{U}$ of Example 3.2.

366 adjacency matrix of a digraph $\mathcal{D}=\left\langle V_{u}, E_{u}\right\rangle$ with $V_{u}=Q \cup \mathfrak{Q}$ and the set $E_{u}$ represents the arcs of which the elements of the matrix $\boldsymbol{U}$ represent the probability that they are covered.

Example 3.2. The digraph of a matrix $\boldsymbol{U}$ of four states three of which, $q_{1}, q_{2}, q_{3}$, belong to the set $Q$ and one, $\mathfrak{q}_{1}$, belongs to the set $\mathfrak{Q}$, is represented by:

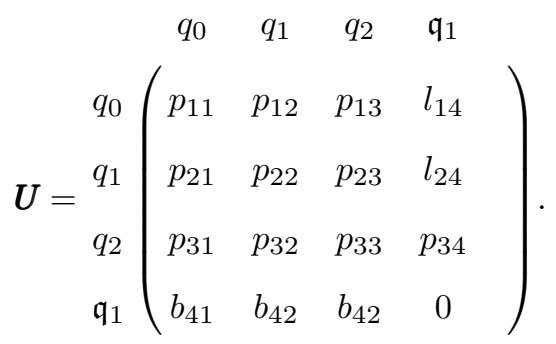

${ }_{371}$ For $r=3$ the digraph will be:

372 All nodes belonging to the set $Q$ of the states are accessible and communicating 373 with each other according to the values assumed by the inputs of the matrix $\boldsymbol{U}$. ${ }_{374}$ Each state can be determined based on the values assumed by the elements of 
the matrix $U$

376 According to Def. (13) all the nodes belonging to the set $\mathfrak{Q}$, are neither accessible 377 nor communication between one another.

Proposition 2. All states belonging to the set $Q$ are absorbent if the state of 379 the node that precedes them belongs to $\mathfrak{Q}$.

Proof. This follows from the Def. (19) and from Eq. 22. Moreover, the fact

381

382 to $\mathfrak{Q}$ has one and only one absorbing state in the set $Q$.

383

Proposition 3. All the states belonging to the set $\mathfrak{Q}$ are transitory states.

385

386 Eq. 10. For this reason the elements of the matrix $U$, that lead from $\mathfrak{Q}$ to $\mathfrak{Q}$

387 are null.

388

з89 3.5 Stochastic matrix of the alphabet $\Gamma$

390 Consider the Lemma (1).

391 Given a probability space $\left(\Omega_{1}, \mathcal{F}_{1}, \mathbb{P}_{1}\right)$. At each probability value of matrix

${ }_{392} \boldsymbol{P}_{\Gamma}$ the probability of an incompatible event can be added. In order to make 393 the transition probabilities vary, also in this case a null square matrix is de394 fined: 


\section{Definition 13.}

$$
\boldsymbol{S}=\left(\begin{array}{cccc}
q_{11} & q_{12} & \ldots & q_{1 m} \\
q_{21} & q_{22} & \ldots & 2 m \\
\vdots & \vdots & \ddots & \vdots \\
q_{m 1} & q_{m 2} & \ldots & q_{m m}
\end{array}\right) \quad \text { with } \quad q_{11}=q_{1 m}=\cdots=q_{m m}=0
$$

One obtains that the set $\mathfrak{S}$ :

$$
\mathfrak{S} \notin \Gamma \quad \text { and } \quad \mathfrak{S} \notin A .
$$

Making the direct sum $\boldsymbol{V}=\boldsymbol{P}_{\Gamma} \oplus \boldsymbol{S}$ one obtains a square matrix:

$$
\boldsymbol{V}=\left(\begin{array}{cccccccc}
p_{11} & p_{12} & \ldots & p_{1 r} & 0 & 0 & \ldots & 0 \\
p_{21} & p_{22} & \ldots & p_{2 r} & 0 & 0 & \ldots & 0 \\
\vdots & \vdots & \ddots & \vdots & 0 & 0 & \ldots & 0 \\
p_{r 1} & p_{r 2} & \ldots & p_{r r} & 0 & 0 & \ldots & 0 \\
0 & 0 & \ldots & 0 & q_{11} & q_{12} & \ldots & q_{1 m} \\
0 & 0 & \ldots & 0 & q_{21} & q_{22} & \ldots & 2 m \\
\vdots & \vdots & & \vdots & \vdots & \vdots & \ddots & \vdots \\
0 & 0 & \ldots & 0 & q_{m 1} & q_{m 2} & \ldots & q_{m m}
\end{array}\right)
$$

398 Also in this case the defined matrix can be divided into four blocks:

$$
V=\left(\begin{array}{c|c}
P_{\Gamma} & N \\
\hline O & S
\end{array}\right) .
$$

399 The submatrix $\boldsymbol{P}_{\Gamma}$ and the submatrix $\boldsymbol{S}$ have already been defined, while the 
400 submatrices $\boldsymbol{O}$ and $\boldsymbol{N}$ are modified by the following

\section{Definition 14.}

$$
\boldsymbol{O}=\left(\begin{array}{cccc}
c_{(r+1) 1} & c_{(r+1) 2} & \ldots & c_{(r+1) r} \\
c_{(r+2) 1} & c_{(r+2) 2} & \ldots & c_{(r+2) r} \\
\vdots & \vdots & \ddots & \vdots \\
c_{(r+m) 1} & c_{(r+m) 2} & \ldots & c_{(r+m) r}
\end{array}\right)
$$

Where, for each $i \in \mathbb{N}_{r+m}$ and $j \in \mathbb{N}_{r}$ one has:

$$
\left(c_{i j}\right)=\{0,1\} \quad \text { and } \quad \sum_{j=1}^{r} c_{i j}=1 .
$$

${ }_{401}$ The $\boldsymbol{N}$ block is modified and becomes a matrix that contains the transition 402 probabilities that link the characters of the alphabet to the transition code.

${ }_{403}$ The following definition is given

Definition 15.

$$
\boldsymbol{N}=\left(\begin{array}{cccc}
g_{1(r+1)} & g_{1(r+2)} & \ldots & g_{1(r+m)} \\
g_{2(r+1)} & g_{2(r+1)} & \ldots & g_{2(r+m)} \\
\vdots & \vdots & \ddots & \vdots \\
g_{r(r+1)} & g_{r(r+2)} & \ldots & g_{r(r+m)}
\end{array}\right)
$$

One set the $V$ matrix is a stochastic matrix for each $i \in \mathbb{N}_{r}$ and $j \in \mathbb{N}_{r+m}$ must be:

$$
\sum_{j=1}^{r} p_{i j}+\sum_{j=r+1}^{m} p_{i j}=1 .
$$

Then substituting the submatrix $\boldsymbol{N}$ in the block the of stochastic matrix $\boldsymbol{V}$ one 
obtains the stochastic matrix of the alphabet ${ }^{9}$ :

$$
\boldsymbol{V}=\left(\begin{array}{cccccccc}
p_{11} & p_{12} & \ldots & p_{1 r} & g_{1(r+1)} & g_{1(r+2)} & \ldots & g_{1(r+m)} \\
p_{21} & p_{22} & \ldots & p_{2 r} & g_{2(r+1)} & g_{2(r+1)} & \ldots & g_{2(r+m)} \\
\vdots & \vdots & \ddots & \vdots & \vdots & \vdots & & \vdots \\
p_{r 1} & p_{r 2} & \ldots & p_{r r} & g_{r(r+1)} & g_{r(r+2)} & \ldots & g_{r(r+m)} \\
c_{(r+1) 1} & c_{(r+1) 2} & \ldots & c_{(r+1) r} & 0 & 0 & \ldots & 0 \\
c_{(r+2) 1} & c_{(r+2) 2} & \ldots & c_{(r+2) r} & 0 & 0 & \ldots & 0 \\
\vdots & \vdots & & \vdots & \vdots & \vdots & \ddots & \vdots \\
c_{(r+m) 1} & c_{(r+m) 2} & \ldots & c_{(r+m) r} & 0 & 0 & \ldots & 0
\end{array}\right)
$$

404 The modified $\boldsymbol{V}$ matrix is still a stochastic matrix. Moreover this matrix represents the adjacency matrix of a digraph $\mathcal{D}=\left\langle V_{v}, E_{v}\right\rangle$ with $V_{v}=\Gamma \cup \mathfrak{S}$, where the set $E_{v}$ includes the arcs whose probability to be covered is represented by the elements of the matrix $\boldsymbol{V}$.

Example 3.3. One considers the following matrix $\boldsymbol{V}_{1}$, with $s_{1}, s_{2} \in \Gamma$ and $\mathfrak{s}_{1} \in \mathfrak{S}$.

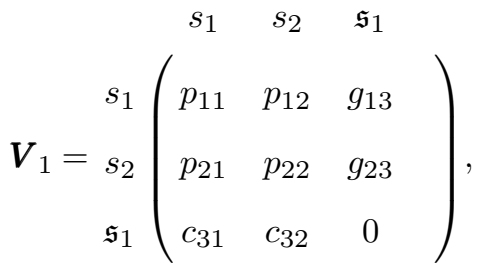

${ }_{408}$ for $r=2$ one has the digraph in Fig. 2:

${ }_{409} \quad 3.6$ Stochastic displacement matrix $D$

${ }_{410}$ A TM reads and writes on a one-dimensional tape on which it is necessary to 411 move the read-write head, so that the possible degrees of freedom are the three ${ }_{412}$ displacements $D=\{d, i, S\}$, and this set must be contained in a PM, according ${ }_{413}$ to Eq. (14). The set $D$, however, must be represented by a Markov matrix, as

\footnotetext{
${ }^{9}$ This stochastic matrix will be studied in the physical part of this text
} 


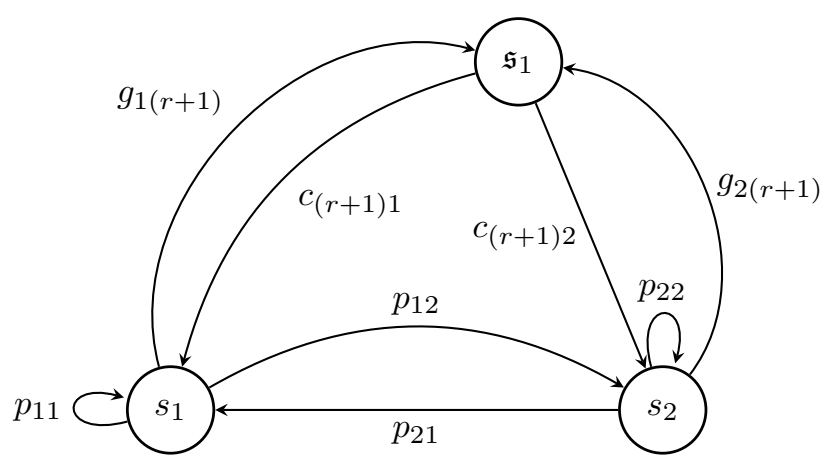

Figure 2: Digraph of matrix $\boldsymbol{V}_{1}$ in Example 3.3.

414 it follows from the arguments used for $U$ and $V$ above.

415 Definition 16. Consider the lemma (1). Given a probability space $\left(\Omega_{2}, \mathcal{F}_{2}, \mathbb{P}_{2}\right)$, 416 one defines a matrix:

$$
D=\left(\begin{array}{cccc}
p_{11} & p_{12} & h_{13} & h_{14} \\
p_{21} & p_{22} & h_{23} & h_{24} \\
d_{31} & d_{32} & 0 & 0 \\
d_{41} & d_{42} & 0 & 0
\end{array}\right) .
$$

${ }_{417}$ A set $\mathfrak{D} \notin D$, which does not represent any real displacement, but a change in 418 the direction of the head of the PM.

It should be noted that the Markov matrix $\boldsymbol{D}$ will give values that do not belong to the set $\{d, s\}$ i.e., values for which a true displacement can not be defined. Then one has the following definition:

Definition 17. Given a Markov matrix $D$, all the values such that the components of the matrix $\boldsymbol{D}$ do not yield a movement $\{d, s\}$ (that is for the set of matrices for which $\left.\overline{\mathbf{D}}=\left\{P_{D}|i=j| p_{i j} \neq 1\right\}\right)$ are not interpreted by a TM, and they hold the TM in a position $s \in S$.

Therefore, the fact that the head of a TM remains fixed in a position of the writing-reading tape is determined by an interpretation of the stochastic matrix $D$. If the configurations $r$ or $l$ are clearly determinable the PM will memorize a 


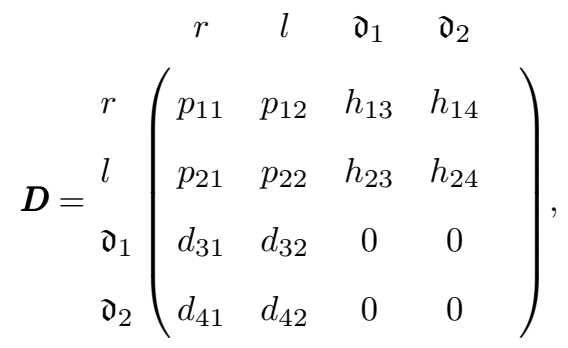

${ }_{432}$ with $\mathfrak{d}_{1}, \mathfrak{d}_{2} \in \mathfrak{D}$.

So the ordered tuple:

$$
\left\langle\mathbf{p}_{U}^{(l)}, \mathbf{p}_{V}^{(m)}, \mathbf{p}_{D}^{(n)}\right\rangle, \quad \text { with } \quad l, m, n \in \mathbb{N}
$$

${ }_{441}$ represents the initial distribution of the system. The product of these stochastic

${ }_{442}$ vectors by the respective stochastic matrices determines the symbol, status and

443 direction exiting a computation. There will be, for a given initial distribution

444 of the three stochastic vectors defined by Eq. (32): 


$$
\begin{aligned}
& \mathbf{p}_{U}^{(0)} \cdot \boldsymbol{U}=\mathbf{p}_{U}^{(1)} \\
& \mathbf{p}_{V}^{(0)} \cdot \boldsymbol{V}=\mathbf{p}_{V}^{(1)} \\
& \mathbf{p}_{D}^{(1)} \cdot \boldsymbol{D}=\mathbf{p}_{D}^{(1)} .
\end{aligned}
$$

${ }_{445}$ So the tuple of the three stochastic vectors $\left\langle\mathbf{p}_{U}^{(1)}, \mathbf{p}_{V}^{(1)}, \mathbf{p}_{D}^{(1)}\right\rangle$ expresses respec-

446 tively a state, a symbol and a direction usable by an PM.

Example 3.4. One considers the following matrices:

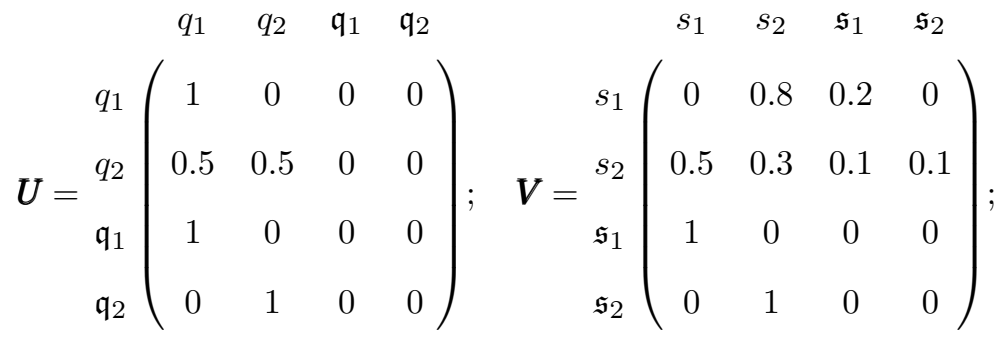

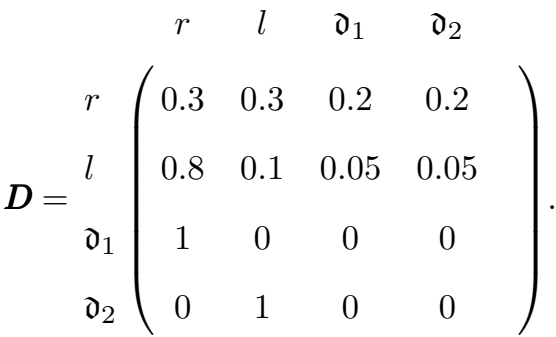

The tuple representing the initial probability distribution is:

$$
\begin{aligned}
& \mathbf{p}_{U}^{(0)}=\left(\begin{array}{llll}
0,1 & 0,2 & 0,4 & 0,3
\end{array}\right) ; \quad \mathbf{p}_{V}^{(0)}=\left(\begin{array}{llll}
0,6 & 0,2 & 0,1 & 0,1
\end{array}\right) ; \\
& \mathbf{p}_{D}^{(0)}=\left(\begin{array}{llll}
1 & 0 & 0 & 0
\end{array}\right) .
\end{aligned}
$$

By multiplying for the respective matrices $\boldsymbol{U}, \boldsymbol{V}$ and $\boldsymbol{D}$, the following stochastic 
vectors are obtained:

$$
\begin{array}{lll}
\mathbf{p}_{U}^{(1)}=\left(\begin{array}{llll}
0,6 & 0,4 & 0 & 0
\end{array}\right) & \in K \cup \mathfrak{Q} ; \\
\mathbf{p}_{V}^{(1)}=\left(\begin{array}{llll}
0,2 & 0,64 & 0,14 & 0,02
\end{array}\right) & \in A \cup \mathfrak{S} ; \\
\mathbf{p}_{D}^{(1)}=\left(\begin{array}{llll}
0,3 & 0,3 & 0,2 & 0,2
\end{array}\right) & \in D \cup \mathfrak{D} .
\end{array}
$$

\section{3.8 On the variation of the Markov stochastic process}

${ }_{448}$ The PM is defined by Markov stochastic processes, described by the matrices $\boldsymbol{U}$,

${ }_{449} \boldsymbol{V}$ and $\boldsymbol{D}$. If these matrices would remain the same at each step of computation,

${ }_{450}$ one would obtain a homogeneous stochastic processes. However, in fact the prob-

451 abilities of the events may change at each step, and so should do the matrices

${ }_{452} U, V$ and $\boldsymbol{D}$. Therefore the following proposition is to be considered:

Proposition 4. The difference between two square stochastic matrices of order $n$, generates a matrix $W$ such that:

$$
W=\left(w_{i j}\right), \quad i, j=1,2, \ldots, n \in \mathbb{N}
$$

with

$$
-1 \leq w_{i j} \leq 1 \quad \forall \quad i, j=1,2, \ldots, n \in \mathbb{N}
$$

Furthermore, for each line it must be verified that

$$
\sum_{i=1}^{n} w_{i j}=0 \text {. }
$$

Proof. Let $\boldsymbol{A}=a_{i j}$ and $\boldsymbol{B}=b_{i j}$ be two stochastic matrices. For the first condition one has that the elements $a_{i j}$ of the stochastic matrix $\boldsymbol{A}$, vary within the interval $[0,1]$ while, because of the difference

$$
A-B
$$


${ }_{453}$ the elements of the matrix $-\boldsymbol{B}$ will vary within the interval $[0,-1]$. So the set ${ }_{454}$ given by $a_{i j}-b_{i j}$ must vary within the interval $[-1,1]$.

Regarding the second condition, considering that for each row of each matrix one must have:

$$
\sum_{i=1}^{n} a_{i j}=1 \quad \text { and } \quad \sum_{i=1}^{n} b_{i j}=1
$$

If one considers the first line of each of the two matrices, one has for $\boldsymbol{A}$ :

$$
\sum_{i=1}^{n} a_{i 1}=a_{11}+a_{21}+a_{31}+\cdots+a_{i 1}=1
$$

Equally for $B$ :

$$
\sum_{i=1}^{n} b_{i 1}=b_{11}+b_{21}+b_{31}+\cdots+b_{i 1}=1
$$

Then the difference:

$$
\sum_{i=1}^{n} a_{i 1}-\sum_{i=1}^{n} b_{i 1}=a_{11}-b_{11}+a_{21}-b_{21}+a_{31}-b_{31}+\cdots+a_{i 1}-b_{i 1}=1-1=0 .
$$

${ }_{455}$ The same reasoning can be repeated for each row of $\boldsymbol{A}$ and $\boldsymbol{B}$.

${ }_{457}$ In order to let the content of one or more elements of the matrices $U, V$ and $D$

458 vary, the following definitions are given at each computation step.

Definition 18. One defines a matrix $W_{U}$ :

$$
\boldsymbol{W}_{U}=\boldsymbol{U}_{a}-\boldsymbol{U}_{b}=\left(\begin{array}{cccc}
w_{11} & w_{12} & \ldots & w_{1 n} \\
w_{21} & w_{22} & \ldots & w_{2 n} \\
\vdots & \vdots & \ddots & \vdots \\
w_{n 1} & w_{n 2} & \ldots & w_{n n}
\end{array}\right)
$$

${ }_{459}$ Repeating the same reasoning for $\boldsymbol{V}$ and $\boldsymbol{D}$ one has: 
Definition 19. One defines a matrix $\boldsymbol{W}_{V}$ :

$$
W_{V}=V_{a}-V_{b} .
$$

Definition 20. One defines a matrix $W_{D}$ :

$$
W_{D}=D_{a}-D_{b}
$$

460 According to Proposition (4) any $\boldsymbol{W}$ matrix can be obtained as the difference between at least two stochastic matrices of the same order.

Definition 21. Adding a generic stochastic matrix $\boldsymbol{A}=\left(a_{i j}\right)$ to a $\boldsymbol{W}=\left(w_{i j}\right)$ matrix of the same order $n$ one again obtains a stochastic matrix if and only if the following conditions are met:

$$
\begin{aligned}
& \sum_{j=1}^{n} a_{i j}+w_{i j}=1 ; \\
& \sum_{j=1}^{n} w_{i j}=0 ; \\
& a_{i j}+w_{i j} \quad \in[0,1] \quad \forall a_{i j}, w_{i j} \in \mathbb{R} .
\end{aligned}
$$

465 The generic matrix $\boldsymbol{W}$ is able to modify the transition probabilities of the ma${ }_{466}$ trices $\boldsymbol{U}, \boldsymbol{V}, \boldsymbol{D}$ at each computation step. Obviously the $\boldsymbol{W}$ matrix can also be ${ }_{467}$ null. The justification for its construction is obtained by taking into account ${ }_{468}$ the axioms of the probability theory (Kolmogorov 1956), discussed in Section 469 (1) below.

Denote by $\boldsymbol{A}^{(t)}$ and $\boldsymbol{W}^{(t)}$ respectively a stochastic matrix and a matrix $\boldsymbol{W}$ with an index $t \geq 0$. Moreover, let $\mathbf{p}^{(0)}$ be the initial distribution of a family of random variable $X_{t}$ belonging to a set of finite $S$ states, defined on a probability space $(\Omega, \mathcal{F}, \mathbb{P})$. Then a homogeneous Markov chain, given the initial distribution $\mathbf{p}^{(0)}$, for $t$ steps will be given by the following of Chapman-Kolmogorov 
equation:

$$
\mathbf{p}^{(t)}=\mathbf{p}^{(0)}\left(\boldsymbol{A}^{(t)}+\boldsymbol{W}^{(t)}\right)^{t},
$$

with the conditions

$$
\begin{aligned}
& \sum_{j=1}^{n} a_{i j}^{(t)}+w_{i j}^{(t)}=1 ; \\
& a_{i j}+w_{i j} \quad \in[0,1] \quad \forall a_{i j}, w_{i j} \in \mathbb{R} .
\end{aligned}
$$

By considering in particular the matrices $\boldsymbol{U}, \boldsymbol{V}, \boldsymbol{D}$ e and the respective matrices $\boldsymbol{W}_{U}, \boldsymbol{W}_{V}, \boldsymbol{W}_{D}$, one defines three initial distributions on the sets $Q \cup$ $\mathfrak{Q}, \Gamma \cup \mathfrak{S}, D \cup \mathfrak{D}$. The initial distributions defined in Eq. (32), are the vectors $\mathbf{p}_{U}^{(0)}, \mathbf{p}_{V}^{(0)}, \mathbf{p}_{D}^{(0)}$. one has then that:

$$
\left\{\begin{array}{l}
\mathbf{p}_{U}^{(l)}=\mathbf{p}_{U}^{(0)} \cdot\left(\boldsymbol{U}^{(0)}+\boldsymbol{W}_{U}^{(0)}\right) \cdot\left(\boldsymbol{U}^{(1)}+\boldsymbol{W}_{U}^{(1)}\right) \cdot \ldots \cdot\left(\boldsymbol{U}^{(l)}+\boldsymbol{W}_{U}^{(l)}\right) \\
\mathbf{p}_{V}^{(m)}=\mathbf{p}_{V}^{(0)} \cdot\left(\boldsymbol{V}^{(0)}+\boldsymbol{W}_{V}^{(0)}\right) \cdot\left(\boldsymbol{V}^{(1)}+\boldsymbol{W}_{V}^{(1)}\right) \cdot \ldots \cdot\left(\boldsymbol{V}^{(m)}+\boldsymbol{W}_{V}^{(m)}\right) \\
\mathbf{p}_{D}^{(n)}=\mathbf{p}_{D}^{(0)} \cdot\left(\boldsymbol{D}^{(0)}+\boldsymbol{W}_{D}^{(0)}\right) \cdot\left(\boldsymbol{D}^{(1)}+\boldsymbol{W}_{D}^{(1)}\right) \cdot \ldots \cdot\left(\boldsymbol{D}^{(n)}+\boldsymbol{W}_{D}^{(n)}\right),
\end{array}\right.
$$

where:

$$
\begin{aligned}
& \boldsymbol{U}^{(l)}=\boldsymbol{U}^{(l-1)}+\boldsymbol{W}_{U}^{(l-1)} \\
& \boldsymbol{V}^{(m)}=\boldsymbol{V}^{(m-1)}+\boldsymbol{W}_{V}^{(m-1)} \\
& \boldsymbol{D}^{(n)}=\boldsymbol{D}^{(n-1)}+\boldsymbol{W}_{D}^{(n-1)} .
\end{aligned}
$$

In conclusion, three independent stochastic processes give rise to three stochastic vectors. Furthermore the generated Markov processes are not homogeneous if the matrices $\boldsymbol{W}_{Q}, \boldsymbol{W}_{V}, \boldsymbol{W}_{D}$ are not simultaneously null. To get an idea of this procedure, consider the following example:

Example 3.5. A library consisting of several floors contains a finite number of books in each floor. A professor in the morning before opening to the public enters the library and accesses a floor via an elevator, but the elevator is faulty, the selected floor may not correspond to the floor where the elevator stops. The teacher marks the plan, accesses it and chooses a book based on the title. It 
${ }_{479}$ also marks the position by using the books to the left and right of the chosen

${ }_{480}$ text as a reference to resume it on the next morning. Immediately after the

${ }_{481}$ professor, a huge group of students entered the library to consult the books and

${ }_{482}$ at the end of the day put the books on the shelves in an order deliberately chosen

${ }_{483}$ by each student. In addition, the librarian often changes the titles of the books

${ }_{484}$ that are not known to all users. At the end of the evening, just before closing,

${ }_{485}$ the professor will return the book taken in the morning to the librarian.

${ }_{486}$ The floor of the library to which the elevator will stop is represented by the ${ }_{487}$ vector $\mathbf{p}_{D}$, the position of the books inside the floor is specified by the vector ${ }_{488} \mathbf{p}_{U}$, while the titles of the books are represented by the vector $\mathbf{p}_{V}$. The behavior ${ }_{489}$ of the elevator is described by a stochastic matrix given by $\boldsymbol{D}^{(n)}+\boldsymbol{W}_{D}^{(n)}$, the ${ }_{490}$ behavior of the students by $\boldsymbol{U}^{(l)}+\boldsymbol{W}_{U}^{(l)}$ and finally the behavior of the librarian ${ }_{491}$ by $\boldsymbol{V}^{(m)}+\boldsymbol{W}_{V}^{(m)}$.

${ }_{492}$ Given an initial distribution of the three vectors, $\left(\mathbf{p}_{U}^{(0)}, \mathbf{p}_{V}^{(0)}, \mathbf{p}_{D}^{(0)}\right)$ represents an ${ }_{493}$ initial probability distribution of: the arrangement of books within the library, 494 the titles of the books that are present, the floor to which the elevator stops ${ }_{495}$ by pushing a botton, respectively. Therefore, with this initial distribution it is 496 possible to know the probability distributions of the three stochastic vectors at 497 each successive access of the professor to the library.

${ }_{498}$ As the example underlines, the state of the system is described by three stochas${ }_{499}$ tic processes, an initial distribution and three successive vectors $\left(\mathbf{p}_{U}^{(l)}, \mathbf{p}_{V}^{(m)}, \mathbf{p}_{D}^{(n)}\right)$ ${ }_{500}$ after $l, m, n$ steps of each stochastic process.

501 The probability of finding the book chosen by the professor and returned to the 502 librarian will be discussed abstractly in Section 6 . 
Proof. For $n, m \in \mathbb{N}$ one has:

$$
P_{U}=\left\{\boldsymbol{U}_{1}, \boldsymbol{U}_{2}, \ldots, \boldsymbol{U}_{n}\right\} \quad \text { and } \quad P_{V}=\left\{\boldsymbol{V}_{1}, \boldsymbol{V}_{2}, \ldots, \boldsymbol{V}_{m}\right\}
$$

Moreover one has:

$$
\begin{aligned}
& Q \subset K \subset P_{U}, \\
& \bar{\Gamma} \subset A \subset P_{V} .
\end{aligned}
$$

${ }_{518}$ Then one assumes that a TM can indifferently work with one of the three sets

519 of the states $Q, K, P_{U}$ and with one of the sets of the alphabet $\bar{\Gamma}, A, P_{V}$. On the ${ }_{520}$ contrary, a PM is able to work only with the sets of $K, P_{U}$ and one of the sets ${ }_{521}$ of the alphabet $A, P_{V}$. 
Therefore, for a PM the instant configuration $\mathcal{C}^{\prime}$ is given:

$$
\mathcal{C}^{\prime}=X^{\prime} \circ P_{Q} \circ Y^{\prime}
$$

with:

${ }^{524} \quad(1) X^{\prime} \in P_{V} \circ P_{V}^{*}$;

525

(2) $P_{Q} \in P_{U}$;

${ }_{526} \quad(3) Y^{\prime} \in P_{V}^{*} \circ P_{V}$.

The configuration $\mathcal{C}^{\prime}$ represents a complete instant configuration of a PM. A PM which carries out any computation, which will be indicated by the sign $\vdash$, passes through a series of instantaneous configurations $c_{i}^{\prime}$ and is indicated:

$$
\cdots \vdash c_{i}^{\prime} \vdash c_{1}^{\prime} \vdash c_{2}^{\prime} \vdash \cdots \vdash c_{j}^{\prime} \vdash \ldots
$$

Observation 3.4. A PM, in its definition set, possesses initial states $q_{0}$ and final states $F$ but they are recognized as such only by a TM. All internal states of a PM are equivalent for a PM.

530 If a computation is performed again with the same steps there will be a proba-

531 bility that it will give the same result from $c_{i}^{\prime}$ to $c_{j}^{\prime}$.

532 Less formally, the PM contains in itself all the elements recognized by a TM, but

${ }_{533}$ they are scattered within a set of random elements that must be focused in order

${ }_{534}$ to be recognized by a TM. A TM recognizes well-defined internal states and

${ }_{535}$ alphabet, thus representing stochastic matrices whose components give almost

536 certain values.

${ }_{537}$ The $\boldsymbol{U}$ and $\boldsymbol{V}$ matrices may contain absorbent elements, whose values are almost 
${ }_{538}$ certainly close to one. This means that these states are recurring. This also 539 means each symbol according to the Definition (4), recognized by a TM is 540 determined by the values assumed by the components of the matrix.

Example 3.6. Given an alphabet of four symbols $s_{1}, s_{2}, s_{3}, s_{4}$ belonging to $\Gamma$ and of four elements $\mathfrak{s}_{1}, \mathfrak{s}_{2}, \mathfrak{s}_{3}, \mathfrak{s}_{4}$ belonging to $\mathfrak{S}$. The matrix $\boldsymbol{V}$ representing a symbol may be the following:

$$
\boldsymbol{V}=\left(\begin{array}{cccccccc}
0.3 & 0.2 & 0.2 & 0.3 & 0 & 0 & 0 & 0 \\
0.3 & 0.2 & 0.1 & 0.4 & 0 & 0 & 0 & 0 \\
0.3 & 0.3 & 0.2 & 0.2 & 0 & 0 & 0 & 0 \\
0.4 & 0.2 & 0.2 & 0.2 & 0 & 0 & 0 & 0 \\
1 & 0 & 0 & 0 & 0 & 0 & 0 & 0 \\
0 & 1 & 0 & 0 & 0 & 0 & 0 & 0 \\
0 & 0 & 1 & 0 & 0 & 0 & 0 & 0 \\
0 & 0 & 0 & 1 & 0 & 0 & 0 & 0
\end{array}\right)
$$

If one adds to this matrix a $\boldsymbol{W}_{V}$ matrix $\boldsymbol{V}$ is modified and this can make a symbol readable:

$$
\boldsymbol{W}_{V}=\left(\begin{array}{cccccccc}
0.7 & -0.2 & -0.2 & -0.3 & 0 & 0 & 0 & 0 \\
-0.3 & 0.8 & -0.1 & -0.4 & 0 & 0 & 0 & 0 \\
-0.3 & -0.3 & -0.2 & 0.8 & 0 & 0 & 0 & 0 \\
-0.4 & -0.2 & -0.2 & 0.8 & 0 & 0 & 0 & 0 \\
0 & 0 & 0 & 0 & 0 & 0 & 0 & 0 \\
0 & 0 & 0 & 0 & 0 & 0 & 0 & 0 \\
0 & 0 & 0 & 0 & 0 & 0 & 0 & 0 \\
0 & 0 & 0 & 0 & 0 & 0 & 0 & 0
\end{array}\right) .
$$


Then adding up according to the Eq. (19):

$$
\boldsymbol{V}+\boldsymbol{W}_{V}=\left(\begin{array}{cccccccc}
1 & 0 & 0 & 0 & 0 & 0 & 0 & 0 \\
0 & 1 & 0 & 0 & 0 & 0 & 0 & 0 \\
0 & 0 & 1 & 0 & 0 & 0 & 0 & 0 \\
0 & 0 & 0 & 1 & 0 & 0 & 0 & 0 \\
1 & 0 & 0 & 0 & 0 & 0 & 0 & 0 \\
0 & 1 & 0 & 0 & 0 & 0 & 0 & 0 \\
0 & 0 & 1 & 0 & 0 & 0 & 0 & 0 \\
0 & 0 & 0 & 1 & 0 & 0 & 0 & 0
\end{array}\right) .
$$

Given an initial stochastic vector $\mathbf{p}_{V}^{(0)}=(0,0,1,0,0,0,0,0)$, one has:

$$
\mathbf{p}_{V}^{(0)} \cdot\left(\boldsymbol{V}+\boldsymbol{W}_{V}\right)=\mathbf{p}_{V}^{(1)}
$$

${ }_{541}$ so that $\mathbf{p}_{V}^{(1)}$ can be used to define the symbol $s_{3}$.

\section{${ }_{542} 4$ Definition of symbol by a TM}

${ }_{543}$ The example reported above allows us to introduce a formal definition of a sym-

544 bol of an alphabet. A further example is proposed. The symbol that represents

${ }_{545}$ the capital letter $\mathrm{A}$ is recognized by everyone both on printed texts and in texts

546 written manually. In fact to obtain this symbol it is necessary to execute an

547 algorithm, but this algorithm is executable in very different ways, (think about

548 the handwriting of each one of us). Yet, in a hand-written text, in most cases

549 one can recognize the letter A. With this example in mind, one introduces the

550 following Definition:

${ }_{551}$ Definition 22. One defines an elementary TM, $\mathcal{M}_{0}$, able to read a symbol of

552 the alphabet and end the computation in a final state keeping the symbol un-

${ }_{553}$ changed. At the end of the computation $\mathcal{M}_{0}$ is able to start a new computation. 


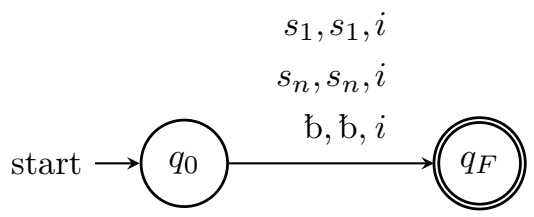

Figure 3: Example of State Transition Graph according to Definition 22 for different symbols.

Observation 4.1. According to Definition (4) if there exists a TM able to terminate a computation starting from a state $q_{0}$ and ending in a state $q_{f}$, so that the computation:

$$
\mathcal{M}_{1}: \quad c_{1} \vdash c_{2} \vdash \cdots \vdash c_{n},
$$

starts always the computation from $\left\langle q_{0}, s_{1}\right\rangle$ and ends the computation always with $\left\langle q_{F}, s_{n}\right\rangle$, then such TM is able to generate a symbol of an alphabet.

For any initial probability distribution of the three vectors

$$
\left(\mathbf{p}_{U}^{(0)}, \mathbf{p}_{V}^{(0)}, \mathbf{p}_{D}^{(0)}\right)
$$

defined in Eq. (32), $\mathcal{M}_{0}$ will always yield in the same value.

Now one assumes that the result obtained by the machine $\mathcal{M}_{1}$ is used by a Universal Turing machine (Turing 1937) $\mathcal{U}=\left\langle Q^{\prime}, \Gamma, \hbar, \delta^{\prime}, q_{0}^{\prime}, F^{\prime}\right\rangle$ for a computation. Therefore, in conclusion, it is possible to obtain the alphabet of a TM if one considers that each symbol can be obtained from an elementary TM and this alphabet can be used by a universal TM.

Proposition 6. For a given alphabet $\Gamma$, consisting of $n$ symbols, there must be at least $n$ initial states $q_{0}^{1}, q_{0}^{2}, \ldots, q_{0}^{n}$ and $n$ final states $f_{1}, f_{2}, \ldots, f_{n}$.

Proof. According to Definition (4) a TM must execute an algorithm to obtain a symbol, thus for $n$ distinguishable symbols must exist $n$ distinct algorithms and therefore $q_{0}^{1}, q_{0}^{2}, \ldots, q_{0}^{n}$ initial states. All symbols must also be recognized by a 
TM, therefore a TM must also terminate the maximal computation, so there 568 must also exist $n$ final unique states.

\section{${ }_{570} 5$ Operation of a PM}

Taking into account the Eqs. (30), (39), (40) and replacing the sets of transition probability matrices in Eqs. (8) and (13) one has:

$$
\mathcal{M}_{\mathcal{P}}=\left\langle P_{U}, \overline{\mathbf{Q}}, P_{V}, B, \zeta, q_{0}, P_{F}\right\rangle .
$$

$$
\zeta:\left(P_{U}-P_{F}\right) \times P_{V} \rightarrow P_{U} \times P_{V} \times P_{D} .
$$

Given a matrix of Markov $U_{0}$, and an initial state $q_{0}$ it must be verified that the path starting from $q_{0}$ through the matrix $U_{0}$ must lead to $q_{0}$ itself, i.e. the path that starts from $q_{0}$, must be absorbing on $q_{0}$. Otherwise the $q_{0}$ state can not be clearly read by a TM interpreting the code. The same holds for any symbol of the alphabet $\Gamma$. To sum up, involved sets are:

$$
\begin{aligned}
& \overline{\mathbf{Q}}=\left\{\bar{q}_{0}, \bar{q}_{1}, \ldots, \bar{q}_{n}\right\}, \\
& Q=\left\{q_{0}, q_{1}, \ldots, q_{n}\right\}, \\
& F=\left\{f_{1}, f_{2}, \ldots, f_{n}\right\},
\end{aligned}
$$




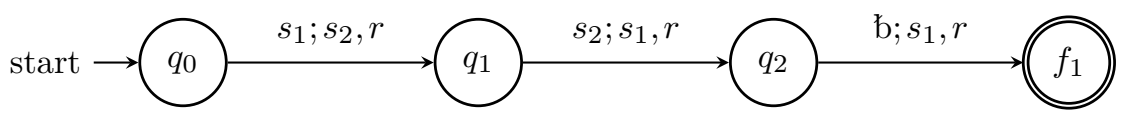

Figure 4: Graph of the program to run from a TM in Tab. 1

and:

$$
\begin{aligned}
B & =\left\{\bar{b}_{1}, \bar{b}_{2}, \ldots, \bar{b}_{n}\right\}, \\
\Gamma & =\left\{s_{1}, s_{2}, \ldots, s_{n}\right\}, \\
\overline{\mathbf{D}} & =\left\{\bar{d}_{0}, \bar{d}_{1}, \ldots, \bar{d}_{n}\right\} .
\end{aligned}
$$

The code generated by a PM is not always readable by a TM, but when this condition occurs, i.e., when a Markov matrix of states has no absorbing states, these values are substituted with the sign $\sqcup$ to indicate a symbol belonging to $\overline{\mathbf{Q}}$ not defined for a TM. Any undefined symbol on a TM can not generate any transition function for a TM, and will be indicated by the abbreviation $n$. $d$. (not determined).

In Table 1 is reported an example of how a PM works. On the left are the values assumed by the definition sets of a PM, given a tuple of initial probability distribution defined in Eq. (32). Then the function $\zeta$ generated by the previous sets appears. Subsequently, the function $\delta$ is presented, which belongs to the $n$-tuple of definition of a TM. The last column of the table presents the interpretation by a TM of the function $\delta$. The symbol $n$. $d$. indicates that a TM does not recognize any function $\delta$ belonging to the definition $n$-tuple.

Points indicate a sequence of instructions that are not defined for a TM.

The described PM executes the instructions using sets not defined for a TM. The first algorithm executable by TM allows it to start the computation on the symbol $s_{1}$. One can be seen graphically in Fig. 4 .

Observation 5.1. The initial configuration of a TM is defined by the sequence: 
Table 1: Example of how a PM works.

\begin{tabular}{llllllll}
$P_{U}$ & $P_{V}$ & $P_{U}$ & $P_{V}$ & $P_{D}$ & $\zeta$ & $\delta$ & TM Resp. \\
\hline$\ldots$ & $\ldots$ & $\ldots$ & $\ldots$ & $\ldots$ & $\ldots$ & $\ldots$ & n. d. \\
$\bar{q}_{1}$ & $s_{2}$ & $q_{1}$ & $s_{2}$ & $\bar{d}_{1}$ & $\zeta\left(\bar{q}_{1}, s_{2}\right)=\left(q_{1}, s_{2}, \bar{d}_{1}\right)$ & $\delta\left(\sqcup, s_{2}\right)=\left(q_{1}, s_{2}, s\right)$ & n. d. \\
$\bar{q}_{2}$ & $\bar{b}_{2}$ & $\bar{q}_{2}$ & $\bar{b}_{3}$ & $\bar{d}_{4}$ & $\zeta\left(\bar{q}_{2}, \bar{b}_{2}\right)=\left(\bar{q}_{2}, \bar{b}_{3}, \bar{d}_{4}\right)$ & $\delta(\sqcup, b)=(\sqcup, b, s)$ & n. d. \\
$q_{1}$ & $\bar{b}_{2}$ & $q_{1}$ & $\bar{b}_{1}$ & $r$ & $\zeta\left(q_{1}, \bar{b}_{2}\right)=\left(q_{1}, \bar{b}_{1}, r\right)$ & $\delta\left(q_{1}, \bar{b}\right)=\left(q_{1}, \bar{b}, r\right)$ & n. d. \\
$\bar{q}_{2}$ & $\bar{b}_{3}$ & $\bar{q}_{2}$ & $\bar{b}_{1}$ & $r$ & $\zeta\left(\bar{q}_{2}, \bar{b}_{3}\right)=\left(q_{1}, \bar{b}_{5}, l\right)$ & $\delta\left(q_{1}, \bar{b}\right)=\left(q_{1}, \bar{b}, l\right)$ & n. d. \\
$q_{0}$ & $s_{1}$ & $f_{1}$ & $s_{2}$ & $r$ & $\zeta\left(q_{0}, s_{1}\right)=\left(f_{1}, s_{2}, r\right)$ & $\delta\left(q_{0}, s_{1}\right)=\left(f_{1}, s_{2}, r\right)$ & \\
$\bar{q}_{2}$ & $s_{1}$ & $f_{1}$ & $s_{3}$ & $l$ & $\zeta\left(\bar{q}_{2}, s_{1}\right)=\left(f_{1}, s_{3}, l\right)$ & $\delta\left(\sqcup, s_{1}\right)=\left(f_{1}, s_{3}, l\right)$ & n. d. \\
$\bar{q}_{2}$ & $\bar{b}_{3}$ & $q_{1}$ & $s_{3}$ & $l$ & $\zeta\left(\bar{q}_{2}, \bar{b}_{3}\right)=\left(\bar{q}_{2}, \bar{b}_{3}, \bar{d}_{2}\right)$ & $\delta(\sqcup, b))=(\sqcup, b, i)$ & n. d. \\
$\ldots$ & $\ldots$ & $\ldots$ & $\ldots$ & $\ldots$ & $\ldots$ & n. d. \\
$\bar{q}_{2}$ & $\bar{b}_{3}$ & $q_{1}$ & $s_{3}$ & $l$ & $\zeta\left(\bar{q}_{2}, \bar{b}_{3}\right)=\left(\bar{q}_{2}, \bar{b}_{3}, \bar{d}_{2}\right)$ & $\delta(\sqcup, b))=(\sqcup, b, s)$ & n. d. \\
$q_{0}$ & $s_{1}$ & $q_{1}$ & $s_{2}$ & $r$ & $\zeta\left(q_{0}, s_{1}\right)=\left(q_{1}, s_{2}, r\right)$ & $\delta\left(q_{0}, s_{1}\right)=\left(q_{1}, s_{2}, r\right)$ & \\
$q_{1}$ & $s_{2}$ & $q_{2}$ & $s_{1}$ & $r$ & $\zeta\left(q_{1}, s_{2}\right)=\left(q_{2}, s_{1}, r\right)$ & $\delta\left(q_{1}, s_{2}\right)=\left(q_{2}, s_{1}, r\right)$ & \\
$q_{2}$ & $\bar{b}_{1}$ & $f_{1}$ & $s_{1}$ & $l$ & $\zeta\left(q_{2}, \bar{b}_{1}=\left(f_{1}, s_{1}, l\right)\right.$ & $\delta\left(q_{2}, \bar{b}\right)=\left(f_{1}, s_{1}, r\right)$ & \\
$\ldots$ & $\ldots$ & $\ldots$ & $\ldots$ & $\ldots$ & $\ldots$ & $\ldots$ & n. d. \\
\hline
\end{tabular}

$\varepsilon q_{0} s_{1} s_{2} s_{3}$, where $\varepsilon=\mathrm{b} \circ \mathrm{b} \circ \mathrm{b} \circ \cdots \circ \mathrm{b}$. The probability that the sets of the matrices $P_{U}, P_{V}, P_{D}$ allow to express values close to one are low compared to all the values between 0 and 1 that could have, so one can assume that any value $q_{0}$ expressed by the matrix $\boldsymbol{U}$ can be preceded by an empty string $\varepsilon$ long enough to be applied to the initial configuration definition for a TM.

\subsection{Programming machine with memory}

A PM with memory is able to store the code it develops.

Definition 23. Given a PM that produces one or more codes, wich are executable by a TM, the set of such codes will be

$$
T=\left\{\mathcal{M}_{1}, \mathcal{M}_{2}, \ldots, \mathcal{M}_{n}\right\}
$$

Moreover, this set $T$ of TMs can be simulated by a Universal Turing machine $\mathcal{U}$

In conclusion, once a PM produces a program that can be used by a TM, the 
Definition 25. Let

$$
\mathcal{M}_{1}=\left\langle Q, \Gamma, b, \delta, q_{0}, F\right\rangle \quad \text { and } \quad \mathcal{M}_{2}=\left\langle Q^{\prime}, \Gamma, b, \delta^{\prime}, q_{0}, F^{\prime}\right\rangle
$$

\section{Information Theory and stochastic matrices}

A PM generates an algorithm that a TM can read and interpret. Any PM does not provide an algorithm in a unique and reliable way. Each step of an algorithm that is created is subject to an uncertainty given by the stochastic matrices of the transition probabilities $\boldsymbol{U}, \boldsymbol{V}, \boldsymbol{D}$. Since a PM combines these three matrices the result is still subject to uncertainty. However, it is possible to define a measure of the uncertainty of the result obtained from the Cartesian product of the definition sets of a PM.

Definition 24. The set on which the information is measured (see Def. 29), is represented by the transitions of the $\zeta$ function of a PM. The transitions of $\zeta$ function are completely random (see the last part of Sec. 3.1).

As one guesses that there are different algorithms, so one wonders how is it possible to distinguish them if performed by different TMs.

be two MTs. They perform two indistinguishable algorithms (Ausiello et al. 2003) if:
(1) $\delta_{0}=\left\langle q_{0}, a, Q, \bar{\Gamma}^{*}, D\right\rangle$ and $\delta_{0}^{\prime}=\left\langle q_{0}, a, Q^{\prime}, \bar{\Gamma}^{*}, D\right\rangle$ with $a \in \bar{\Gamma}^{*}$;
(2) $\forall y \in \bar{\Gamma}^{*} \circ \Gamma \quad \exists \quad c: \delta\langle q, y\rangle \in F \quad$ and $\quad c^{\prime}: \delta^{\prime}\left\langle q^{\prime}, y\right\rangle \in F^{\prime}$;
(3) $Q \equiv Q^{\prime}$ and $F \equiv F^{\prime}$. 
From the first condition one can see that two algorithms are indistinguishable if they start from the same initial transition function, for example one can not distinguish two algorithms that both to compute from the number three, while one can distinguish them if one starts from three and the other from two.

From the second condition it follows that there must exist a transition function that ends with the same output for $\mathcal{M}_{1}$ and $\mathcal{M}_{2}$. The third condition implies that there should not be a different state between $\mathcal{M}_{1}$ and $\mathcal{M}_{2}$, otherwise the two algorithms would be distinguishable for that state.

Given a Markov matrix $\boldsymbol{U}$ or $\boldsymbol{V}$ and a distribution of starting point vectors, these matrices can also be represented as matrices of the adjacency of a digraph. Whenever this digraph is traveled, it could define a symbol if it meets the conditions involved in Definition (4). The production of a symbol by an algorithm generates a discrete source of information that produces symbols belonging to an alphabet $\Gamma=\left\{s_{0}, s_{1}, \ldots, s_{n}\right\}$. Thus a probability is also defined in the space of the $Q=\left\{q_{0}, q_{1}, \ldots, q_{n}\right\}$. The $Q$ states are a set independent of $\Gamma^{10}$. Each symbol or state is characterized by its probability, i.e. there is a probability that the algorithm that produced a symbol can repeat the computation again and end with the same symbol as output.

A TM is able to perform a computation programmed by a PM if it starts with an initial computation $c_{0}=\varepsilon q_{0} y$, moreover if a TM ends the computation it can set itself indefinitely in the initial state and repeat the computation again.

Definition 26. Given the writing $c=x q y$, one indicates with $\mathcal{L}_{\Gamma} \in \Gamma \circ \bar{\Gamma}^{*} \cup$ $\{\varepsilon\}$ the language of the strings that can appear to the left of the state of a configuration. One indicates with $\mathcal{R}_{\Gamma} \in \bar{\Gamma}^{*} \circ \Gamma \cup\{b\}$ the language of the strings that can appear to the right of the state of a configuration.

\footnotetext{
${ }^{10}$ Imagine a man and a rabbit observing the grass of a meadow, the man could think of mowing it, while the rabbit might think to eat it. The same object observed has different interpretations independent of the object.
} 
A computation that starts at $c_{0}$ and leads to $c_{n}$ is referred to as

$$
c_{0} \vdash c_{1} \vdash c_{2} \vdash \cdots \vdash c_{n} \text {, }
$$

649

Then there will be a set of computations for which, given an initial state defined and almost certain, there is an uncertainty in the termination configuration:

$$
c_{0} \vdash^{*} p_{c_{n}} .
$$


Where the $\vdash^{*}$ symbol indicates the reflexive and transitive closure of $\vdash$ to indicate that there is a finite number of transitions that lead from $c_{0}$ to $c_{n}$. This set is defined as:

$$
G=\left\{c_{0} \vdash * p_{c_{n}} \mid p_{c_{0}}=1 \text { q.c., } p_{c_{n}}=[0,1]\right\} \text {. }
$$

Thus, within the set $E$ defined in Section 2, it will be interesting to calculate the probability of:

$$
P\left(X_{n}=\left\langle f, s_{n}\right\rangle \mid X_{0}=\left\langle q_{0}, s_{1}\right\rangle \quad \text { with } \quad X \in G,\right.
$$

Definition 29. One defines the information on the stochastic variable $X$,

$$
I(X):=-\log _{b} P(X) .
$$

Given an alphabet $\Gamma=\left\{s_{1}, s_{2}, \ldots, s_{n}\right\}$, each symbol will have a probability

$$
P\left(s_{1}\right), P\left(s_{2}\right), \ldots, P\left(s_{n}\right),
$$

Definition 30. One defines the information entropy (Shannon 1948):

$$
H(X):=\sum_{n=1}^{n} P\left(s_{n}\right) I\left(s_{n}\right) .
$$


Information entropy provides a weighted average of the information associated with each symbol. A set of algorithms able to end with an unambiguous sequence of symbols can be used to create a uniquely decipherable code. One therefore defines this code as:

$$
\mathcal{A}=\left\{s_{1}, s_{2}, \ldots, s_{n}\right\} \quad n \in \mathbb{N} .
$$

\section{${ }_{680} \quad 7 \quad$ Information of a source without memory and} with memory

The probability of a memory source of a symbol $s_{j} \in \mathcal{A}$ of Eq. 49 , conditioned by the previous emission of a symbol $s_{i} \in \mathcal{A}$ will be:

$$
P\left(s_{j} \mid s_{i}\right),
$$

the entropy of a symbol $s_{i}$ will therefore be:

$$
H\left(\mathcal{A} \mid s_{j}\right)=\sum_{i} P\left(s_{j} \mid s_{i}\right) \log _{2} \frac{1}{\left.P s_{j} \mid s_{i}\right)} .
$$

It must also result:

$$
H\left(\mathcal{A} \mid s_{i}\right) \leq H(\mathcal{A}),
$$

(Shannon 1948). 


\section{Symbols of an alphabet}

712 Then there must be at least one matrix belonging to $\mathcal{V}$ which ends the compu-

713 tation with the symbol $s \in \mathbf{s}$. For each request given by $\left\langle q_{0}, \mathbf{s}\right\rangle$ to $\mathcal{V}$, there must 
be a pair $\left\langle f, \mathbf{s}^{\prime}\right\rangle$ where $f \in F$.

In conclusion, since $\mathcal{V}$ represents a set of stochastic matrices, a compound symbol must be probabilistic. Furthermore, the information relating to the outgoing symbol must be zero or in any case very close to zero. This as a consequence of the request to have uniqueness from the initial state to the final state. Obviously through symbols derived from others it is possible to construct derived alphabets and subsequently languages.

\section{Physical part. Introduction}

The second part of this paper aims to analyze and describe the physical consequences of the definitions given in the first part. A study of living systems must necessarily fit into an analysis of the physical system in which these are observed.

A living system is necessarily also a thermodynamic system. Some examples are proposed. When one looks at any object at a certain distance or when one looks at a clock to know the time, one is actually comparing at least two thermodynamic systems, one measuring and the other being measured. Such systems will certainly have at least one level of energy in common at the time of measurement. If two or more thermodynamic systems carry out a measurement, there must be a common state that corresponds to the measurement itself. For example, when an observer wants to establish the distance that separates him from an object, he constructs an algorithm that divides this distance into parts of a standard measure accepted by the observer, (meter, light year, etc.). The fundamental characteristic of these measurement algorithms is that they must be repeatable, that is, a second observer in the same conditions as the first one must 
${ }_{763}$ The following discussion will be based on these two considerations:

(1) The observation of any physical system represents a measure made on that system. 
(2) The measurement obtained on a physical system is subject to an observer's interpretation $^{11}$.

Finally, it is necessary to point out that in this article the observer makes a measurement in two different steps:

(1) Initial instant called positioning, where he starts the measurement algorithm.

(2) Final instant called measurement, where the measure actually takes place.

The fact that a measurement is carried out in two different steps implies that there must exist an independent set of logical states, in which at least one initial state and one final state must exist in order to make a measurement of a given quantity. This procedure corresponds to the execution of a computation that can be represented abstractly using a Turing machine.

In the quantum mechanics field, the measure theory is still debated (Bell 1987).

\section{On thermodynamics and energy}

A measurement process in practice always undergoes random errors. These random errors may be due to the complexity of the system one wants to measure or may be intrinsic to the system. Therefore it is assumed that each measure can be represented as a stochastic matrix.

In the mathematical part above, the stochastic matrices were introduced to define the symbol of a code. The observer in the act of observing an object begins the measurement at some instant, but does not know how many initial symbols are contained in the stochastic matrix to which the observation refers. That is, the observer is assumed to know only some of the possible initial symbols. For

\footnotetext{
${ }^{11}$ The observer must be understood in the most general sense possible, at the limit a thermodynamic system.
} 
Example 10.1. One considers a stochastic matrix, and one assumes that the positioning stochastic vector is given by the pair $\left\langle q_{0}, \mathbf{p}_{s}=(1,0,0,0)\right\rangle$. From the product of $\mathbf{p}_{s}$ by a stochastic matrix a vector is obtained:

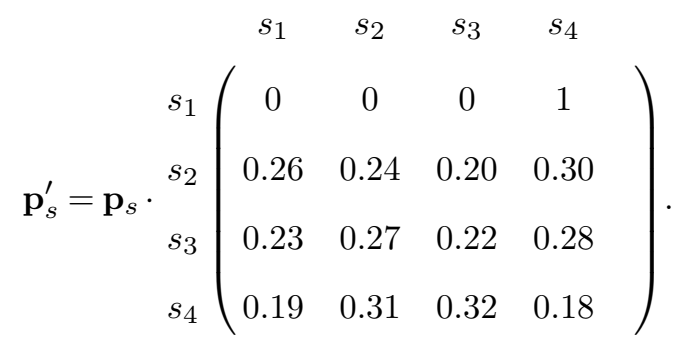

The measurement result returns the triple $\left\langle f, \mathbf{p}_{s}^{\prime}=(0,0,0,1), D\right\rangle$ in which the vector $\mathbf{p}_{s}^{\prime}$ will indicate the symbol observed with greater probability than the others. The states $q_{0}, f$ and the set $D$ represent respectively the initial state, a final state the set of directions of a TM.

Definition 32. One assumes that a TM that operates through an alphabet defined in Eq. (2) whose symbols are defined in Def. (22), a set of states defined in Eq. (6) and a set defined in Def. (16) is a isomorphic model of measure in the physical, chemical and biological fields.

This model operates on a space defined in Def. (53).

About the questions raised on measurement in physics (Bell 1987) one asserts that, in general if it is possible to effect some measures on the Universe, then the set that measures and the measured set they must interact. By Def. (32), this interaction can be represented in an abstract way by assuming that the Measur- 
able Universe can be represented with an ideal tape defined in Def. (1).

This tape is by definition discrete, while the Measurable Universe is not. This problem is overcome by defining in a probabilistic way the symbols of the alphabet through stochastic matrices defined in Eq. 30. In this way every measurable and continuous variation on the Universe corresponds to a measurable and in probabilistic way continuous variation on an ideal tape defined for a TM. Any TM that makes a measurement on this tape must have an initial state for the Def. (1). This assumption allows us to admit that the $q_{0}$ and $f$ logical states, according to Def. (1), can be attributed to a TM that performs the measurement, while they may, not belong to the rest of the Measurable Universe.

Example 10.2. Now let's assume to slide a ball on a $\alpha$ surface, perfectly smooth, so smooth as to be able to imagine it as a surface formed by a single isolated energy level. Now one substitutes the ball with any perturbation localized on the surface. If one wanted to describe at which point of the surface this perturbation is, such measurement would be impossible, since the probability of the perturbation would be the same, instantaneously, for the whole surface. So $\alpha$ surface represents a place where the probability of a perturbation becomes the same everywhere.

However, the presence of a ball or of a perturbation in a point of the surface could be seen as a break of any symmetry of the $\alpha$ energy surface .

This introductory example is thus formalized.

Let $\alpha$ be a hypersurface of constant energy. Let any experiment be given on $\alpha$ that, starting from a point $A$, causes a variation of symmetry on $\alpha$. As a result of the experiment, one expects to have a signal of value $X$ with an associated probability $p(X)$. Let us hypothesize that $A_{m}$ distinguishable and independent experiments on surface $\alpha$ are applicable. One experiment will be distinguishable from another if $A_{i} \neq A_{j}, \quad i, j \in m$. The results of each experiment will be $X_{n}$ with probability $p\left(X_{n}\right)$ representable as a stochastic vector of probability 
distribution

Let us consider the Def. (4) of symbol and assume that any random experiment can be represented by an algorithm. If any symbol is definable by other random experiments, then it is possible to have different symbols corresponding to the results of such random generative experiments. One therefore assumes $n$ distinct symbols. On these $n$ symbols it is possible to construct $n$ distinguishable experiments for the initial symbol $s_{1}, s_{2}, \ldots, s_{n}$. The pair $\left\langle q_{0}, s_{n}\right\rangle$, introduced in Section (1), represents the initial state of each random experiment.

One can imagine that some results of random symbol-generating experiments and some results of random experiments can not be represented as symbols but they are observable because of the effects they have on symbols. For example, an interaction between two bodies is not directly observable, but its effects on bodies are observable. Therefore, it can occur that the results of a random experiment can be modified by the results of other random experiments.

In this Section one wants to show how a Turing machine can represent the abstraction of a work, while a programming machine is the abstraction of a thermal machine ${ }^{12}$.

Let us now consider one of the set of $n$ random experiments and be $\Omega_{1}$ the sample space of all the results of this experiment. Given the probability space $\left(\Omega_{1}, \mathcal{A}, p\right)$ and repeating the same reasoning for each of the $n$ random experiments it is possible to put forward the following Definition:

Definition 33. Let $\alpha$ be a hypersurface of energy. If one performs $n$ random experiments with $\Omega_{1}, \Omega_{2}, \ldots, \Omega_{n}$ sample spaces, a probability space $(\Omega, \mathcal{A}, p)$ is defined for each random experiment. Let the matrix $\boldsymbol{V}_{\alpha}$ as defined in Eq. (30)

\footnotetext{
${ }^{12}$ Both definitions have been given in the mathematical part.
} 
be associated with $\alpha$ :

$$
\boldsymbol{V}_{\alpha}:=\left(\begin{array}{cccccccc}
p_{11} & p_{12} & \ldots & p_{1 r} & g_{1 r+1} & g_{1 r+2} & \ldots & g_{1 r+m} \\
p_{21} & p_{22} & \ldots & p_{2 r} & g_{2 r+1} & g_{2 r+1} & \ldots & g_{2 r+m} \\
\vdots & \vdots & \ddots & \vdots & \vdots & \vdots & & \vdots \\
p_{r 1} & p_{r 2} & \ldots & p_{r r} & g_{r r+1} & g_{r r+2} & \ldots & g_{r r+m} \\
c_{(r+1) 1} & c_{(r+1) 2} & \ldots & c_{(r+1) r} & 0 & 0 & \ldots & 0 \\
c_{(r+2) 1} & c_{(r+2) 2} & \ldots & c_{(r+2) r} & 0 & 0 & \ldots & 0 \\
\vdots & \vdots & & \vdots & \vdots & \vdots & \ddots & \vdots \\
c_{(r+m) 1} & c_{(r+m) 2} & \ldots & c_{(r+m) r} & 0 & 0 & \ldots & 0
\end{array}\right)
$$

$859\left(\Gamma, \mathfrak{S}, I, \boldsymbol{V}_{\alpha}\right)$ where:

\footnotetext{
${ }^{13}$ Terminating means of which it is possible to know the final outcome, as in Ref. (Li and Vitányi 2008; Turing 1937).
} 
The matrix $\boldsymbol{V}_{\alpha}$ is divisible into four blocks, as reported in Eq. (25):

$$
V_{\alpha}=\left(\begin{array}{c|c}
P_{\Gamma} & N \\
\hline O & 0
\end{array}\right),
$$

While the elements of block $\boldsymbol{P}_{\Gamma}$ represents the probability of transition of measurable symbols belonging to an alphabet, the block $N$ represents the distribution of probability of measurable interactions between bodies, the interaction between two bodies can be interpreted as a variation of probability with respect to an initial measure of measurable symbols.

Returning to Example (10.2), if one considers an energy hypersurface $\alpha$, it is expected that all the symbols expressed by the metric associated with $\alpha$, according by Def. (33), have the same average information value. That is, each symbol or interaction has a maximum information, due to the stochasticity of the matrix. Therefore, it is possible to assume that $\alpha$ has a random probability distribution. It is not possible to admit the existence of an associated matrix $\boldsymbol{V}_{\alpha}$ different from $\boldsymbol{V}_{\alpha}$. In other words, $\boldsymbol{V}_{\alpha}$ describes a hypersurface $\alpha$ if given an initial variation on $\alpha$ one has the same uncertainty in the measurement of the symbol or of the output value for all the measurable ones. If one represents $\alpha$ as an algorithm, this algorithm must be stochastic and minimal in the sense that it is not possible to imagine a simpler algorithm that, starting from initial variation on $\alpha$ terminates in the measurement on a symbol or interaction described by $V_{\alpha}$. Incidentally, a proposition formulated by G.J. Chaitin on the randomness of a program, intended as a synonym of algorithm, would seem to connect intuitively energy and algorithms: any minimal program is necessarily random (Chaitin 1990).

Example 10.3. Given a hypersurface $\alpha$ and the related stochastic matrix $\boldsymbol{V}_{\alpha}$ if the identifies a stochastic matrix. The first two columns of $\boldsymbol{V}_{\alpha}$ represent the coding of two symbols $s_{1}$ and $s_{2}$ belonging to an alphabet, for example two bodies, while the following two columns represent interactions $i_{1}$ and $i_{2}$, they can not 
represent an alphabet but allow the transitions between the symbols.

$$
V_{\alpha}={ }_{s_{2}}^{s_{1}} i_{1}\left(\begin{array}{cccc}
s_{1} & s_{2} & i_{1} & i_{2} \\
& i_{2}
\end{array}\left(\begin{array}{cccc}
0,5 & 0 & 0 \\
0,5 & 0,5 & 0 & 0 \\
1 & 0 & 0 & 0 \\
0 & 1 & 0 & 0
\end{array}\right) .\right.
$$

Looking at the matrix $\boldsymbol{V}_{\alpha}$ one notices that it does not clearly express neither $s_{1}$, nor $s_{2}$. Suppose that an interaction intervenes on $\alpha$ precisely on the symbol $s_{2}$ with a probability equal to $p\left(i_{1}\right)=0,5$, then $\boldsymbol{V}_{\alpha}$ must be modified, repeating the sum of the Example (3.6), as follows:

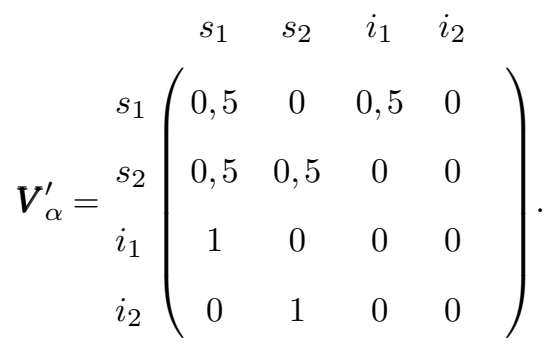

${ }_{890}$ From $V_{\alpha}^{\prime}$ one notes that the symbol $s_{2}$ has zero probability, while $i_{1}$ contributes ${ }_{891}$ to give to the probability of the symbol $s_{1}$ the value one. For $\boldsymbol{V}_{\alpha}$ this is repre892 sented graphically in Fig. 5.

${ }_{893}$ While for the $\boldsymbol{V}_{\alpha}^{\prime}$ is represented graphically in Fig. 6.

${ }^{894}$ One points out that the probability that the final state of the computation is ${ }_{895} s_{1}$ is greater than that of $s_{2}$.

896 An energy hypersurface can be defined as a probabilistic Turing machine where 897 the matrix $V$ represents all the transition functions for possible initial probabil898 ity distribution given by the stochastic vector $\mathbf{p}_{s}$, while the interactions between 899 energy hypersurfaces are represented by modifications of the respective matrices 


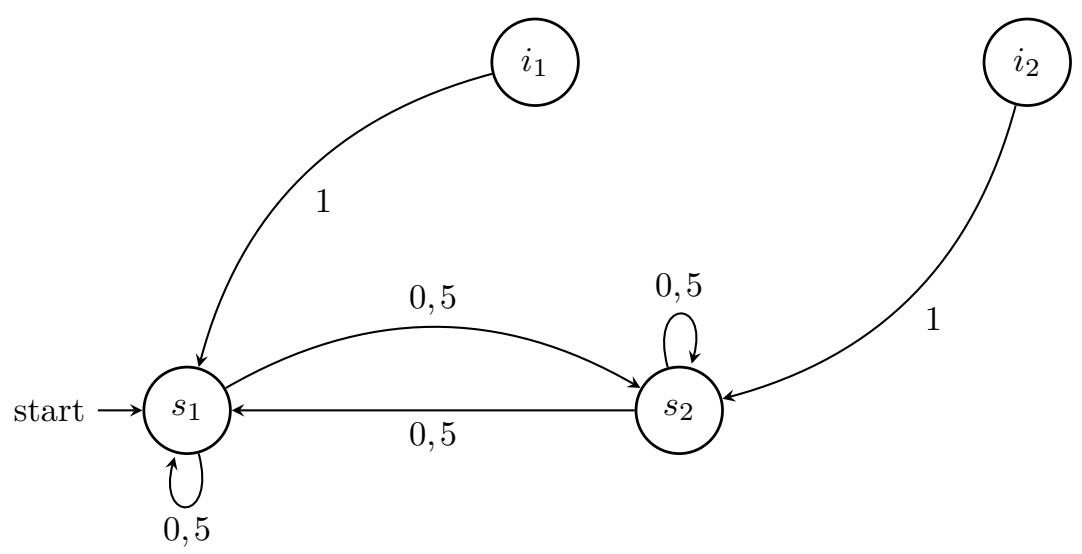

Figure 5: Graph of matrix $\boldsymbol{V}_{\alpha}$ with start point on the symbol $s_{1}$.

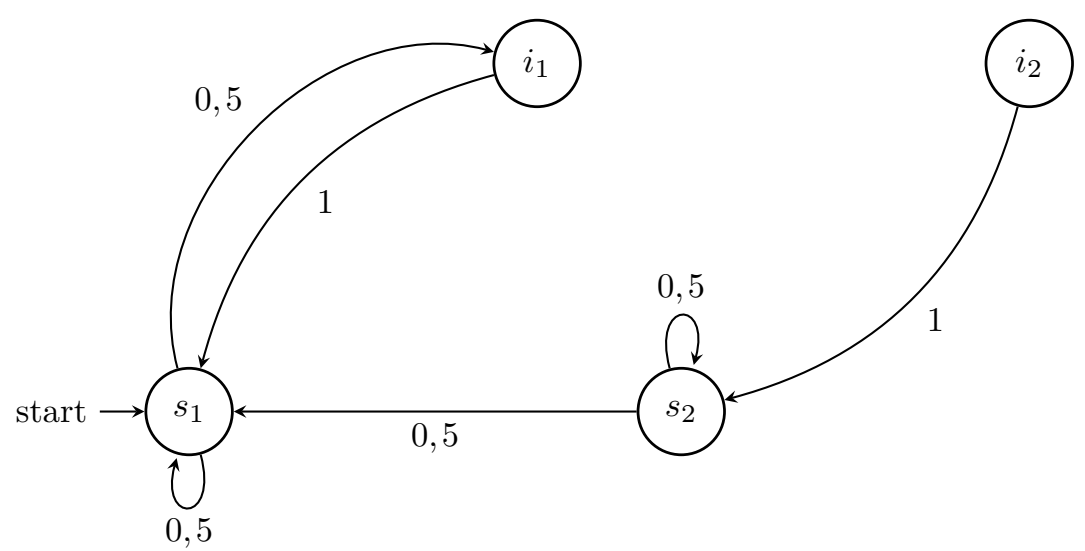

Figure 6: Graph of matrix $\boldsymbol{V}_{\alpha}^{\prime}$ with start point on the symbol $s_{1}$. 
Definition 34. Let $\alpha$ be an energy hypersurface described by

$$
\mathcal{E}_{\alpha}=\left(\Gamma, \mathfrak{S}, I, V_{\alpha}\right)
$$

The matrix that represents a hypersurperfice of energy is not stationary, since the interaction with other hypersurfaces of energy allows variations as described by the Eq. (38). One gives the following

Any perturbation that occurs on $\alpha$ due to the interaction with another hypersurface of energy must determine a variation of some entry of the stochastic matrix associated with $\boldsymbol{V}_{\alpha}$. This perturbation is called variation of symmetry of $\alpha$.

The symmetry variation is observable and therefore measurable as a variation of the state of the system. The experiments on the system will give at least a statistically different result compared to the results before the variation. If two or more hypersurfaces of energy interact the symmetry variations must occur on each of these hypersurfaces. Moreover, changes in the income of matrices associated with energy hypersurfaces can lead to the formation of transient states or recurrent states, they can form communicating states or not.

Consider a thermodynamic system, containing a finite set of energy levels. Let us assume that each energy level retains its symmetry if no external variations occur. In other words, a system in a steady state tends statistically to leave its symmetry unchanged. Threfore, in order to know the whole system it is sufficient to know or measure only its symmetric or antisymmetric portion.

\section{Symmetry variations and constraints to a ther- modynamic operation}


Definition 35 (Thermodynamic operation). Given a probability space

$$
(\Omega, \mathcal{A}, \mu),
$$

922

923

924

if a variation of symmetry on a hypersurface of energy is measurable on this probability space, then this variation of symmetry is defined as thermodynamic operation $O t$.

A $O t$ represents the smallest measurable symmetry variation on a thermodynamic system. A single thermodynamic operation determines at least two symmetry variations on two interacting hypersurfaces of energy. In other words, if the measurable symmetry variation is a signal $S(t)$, it can be assumed that its source is an energy hypersurface $\alpha$ and that the emitted signal is received by the second hypersurface $\beta$. A variation of symmetry determines the transition between a previous state and a subsequent one of the surface. one can define a support $\mathcal{K}=\{\mathcal{O}, \overline{\mathcal{O}}\}$ of an algebra given by the $O t$ and its complementary $\overline{O t}$. Furthermore, two fundamental operations can be defined: union and intersection of states. So the structure $(\mathcal{K}, \cap, \cup)$, represents the smallest $\sigma$-algebra for the set of the two energy hypersurfaces involved in the variation.

Proposition 8 (Termination of an $O t$ ). If an energy hypersurface changes its symmetry, this variation must have a start and end point. The graph corresponds to Def. (22).

Proof. Let $\boldsymbol{V}_{\alpha}$ be the matrix associated with an energy hypersurface, if it varies its symmetry and this variation is measurable with a symbol $s$, the matrix $\boldsymbol{V}_{\alpha}$ must change at least the symmetry of another complementary $s$ symbol so that the definition of stochastic matrix is maintained for the Eq. (29). 


\section{${ }_{944} \quad 11.1$ On thermodynamic systems}

One consider the Definition (7), an initial stochastic vector $\mathbf{p}_{U}$, defined in Eq. (32) and a state spaces defined in Eqs. (9). Given a probability space of the logical states defined in Eq. (6) one has:

$$
\Omega_{1}=\mathbf{p}_{U} \cdot U_{k \geq 0}=\left\{\overline{\mathbf{Q}}, \mathbf{q}_{0}, \mathbf{q}_{1}, \ldots, \mathbf{q}_{n}, F, \mathfrak{Q}\right\}, \quad \forall \quad U_{k} \in P_{U} .
$$

The corresponding probability space is:

$$
\mathcal{R}:\left(\Omega_{1}, \mathcal{F}_{1}, \mathbb{P}_{1}\left(\overline{\mathbf{Q}}, \mathbf{q}_{0}, \mathbf{q}_{1}, \ldots, \mathbf{q}_{n}, F, \mathfrak{Q}\right)\right),
$$

where $\Omega_{1}$ represents the outcome of all the possible experiments given the initial vector $\mathbf{p}_{U}$ and the whole set $P_{U}$. In addition for the alphabet defined in Eq. (30) set, one has:

$$
\Omega_{2}=\mathbf{p}_{V} \cdot V_{h \geq 0}=\left\{\mathfrak{b}, \mathbf{s}_{1}, \mathbf{s}_{2}, \ldots, \mathbf{s}_{n}, \mathfrak{S}\right\}, \quad \forall \quad V_{h} \in P_{V},
$$

\footnotetext{
${ }^{14}$ For example, one can think of a thermal machine, which is theorized as a MT, possessing logical states which can not be measured directly.
} 
and the corresponding probability space is:

$$
\mathcal{S}:\left(\Omega_{2}, \mathcal{F}_{2}, \mathbb{P}_{2}\left(\mathfrak{b}, \mathbf{s}_{1}, \mathbf{s}_{2}, \ldots, \mathbf{s}_{n}, \mathfrak{S}\right)\right)
$$

One finally defines, by Eq. (16) for displacement set, a third probability space:

$$
\Omega_{3}=\mathbf{p}_{D} \cdot \boldsymbol{D}_{j \geq 0}=\{\overline{\mathbf{D}}, \mathbf{l}, \mathbf{r}, \mathfrak{D}\}, \quad \forall \quad \boldsymbol{D}_{j} \in P_{D},
$$

which is related to the probability space:

$$
\mathcal{T}:\left(\Omega_{3}, \mathcal{F}_{3}, \mathbb{P}_{3}(\overline{\mathbf{D}}, \mathbf{l}, \mathbf{r}, \mathfrak{D})\right)
$$

955 Now it is possible to define the product of these probability spaces. iments belong to three different sets. must contain:

965 (1) An initial tuple of three stochastic vectors.

(2) Three stochastic matrices belonging to the sets $P_{U}, P_{V}$ and $P_{D}$.

967 From the product of the stochastic vectors by the respective matrices a new

968 tuple is obtained, which represents the result of the measurement process. one 
Definition 36. The product space

$$
(\Omega, \mathcal{F}, \mathbb{P}),
$$

984 zation.

$$
O=\left\{O t_{1}, O t_{2}, \ldots, O t_{n}\right\},
$$


one defines a thermodynamic algorithm At a concatenation of $O t \in O$ com-

(1) a coding of the transition table of each $O t$;

(3) a tape on which the Ots are stored.

Definition 38. Given a finite set of thermodynamic algorithms

$$
W=\left\{A t_{1}, A t_{2}, \ldots, A t_{n}\right\},
$$

999

one defines a system of thermodynamic operations $S t \subseteq W$.

The following relation is then established:

$$
O t \subseteq S t \subseteq W .
$$

\section{Contraints to an $O t$} probabilities of $\boldsymbol{V}$ vary, the symmetry of the associated energy hypersurface 
varies and the expressions relative to the measurable elements of this matrix vary accordingly. A stochastic matrix with numerous absorbent states determines limits in the variability of the alphabet it expresses, so it is possible that it always determines the same values within $\Gamma$. The information of a stochastic matrix with many adsorbent state is low (see Def. 29 and Ref. (Shannon 1948)). In general, a totally stochastic $S t$ is a thermodynamic system in which every $O t$ that belongs to the system is able to vary its symmetry. In other words, every element that belongs to the system has statistically access to all the energy levels of this system.

\subsection{On the stochastic state of a system of thermody- namic operations}

An St composed of Ots with stochastic matrices whose components possess similar probability measures determines $O t$ s that can result in any symbol. This type of $O t$ s determines totally stochastic assemblies, i. e., within them it is very unlikely to find a symmetry with respect to any symbol, all symbols tend to be with equal probability. This situation obviously lasts as long as the system is not measured because the measurement operation implies that there is a terminal symbol. Such a $S t$ has a high information entropy of its symbols.

In order to represent the stochastic Ots i.e. with high uncertainty on symbols and on $\mathfrak{s} \in \mathfrak{S}$, (which represent interactions), one uses the symbol $O t_{s}$, but this notation does not represent any subset of $O t$. Furthermore, a $S t$ composed of $O t_{s}$ contains stochastic Ats, therefore with a high information entropy. 


\section{${ }_{1028} 13$ Thermodynamic computation}

Given a $S t$, and a pair $\left\langle q_{0}, s\right\rangle$, where $s$ represents any symbol or interaction within $S t$ and $q_{0}$ is the initial state, $\left\langle q_{0}, s\right\rangle$ represents an initial variation of symmetry on St. By considering the definitions of TM one has that the pair $\left\langle q_{0}, s\right\rangle$ can be defined as initial computation $c_{0}$, and the successive steps are indicated with $c_{i} \quad(i=1,2, \ldots, n)$ up to a terminal computation, if it exists. So the execution of a particular At within a $S t$ starts from the pair $c_{0}=\left\langle q_{0}, s\right\rangle$ if it exists:

$$
A t_{1}: \quad c_{0} \vdash c_{1} \vdash c_{2} \cdots \vdash c_{n} \text {. }
$$

If an At defined according to Definition (1) then the execution of any At can be defined as a thermodynamic computation. This computation will end if the state of $c_{n}$ will belong to a final state. Thus, a given terminating $A t$ is described by a table of transition functions, if the last step is of the type $c_{f}:\langle F, \Gamma, D\rangle$, where $F$ is the set of final states. An $A t$ is considered terminated if the computation ends with a final state.

$$
A t: \quad c_{0} \vdash c_{1} \vdash c_{2} \cdots \vdash c_{f} .
$$

The termination of any $A t$ within a $S t$ allows the existence of a symmetry with respect to the computation, i.e. At can be executed again.

Example 13.1. Consider a shepherd, as evidenced in the following Example, counting the sheep of his flock. Every time he finishes the recognition and the count of the sheep, he frees himself and is able to recognize and count again, so he creates a symmetry in the execution of the recognition and counting algorithm.

In general, three different types of computation are created, two of which (i and ii) are called maximal computations:

(1) computations ending in a final state (by convention it is called accepting computation); 
(2) computations that end because a further transition function is not defined (by convention it is called rejecting computation);

(3) non-terminating computation (no response).

Within the first group of accepting computations (all ending with a final state) two sets can be created again:

(a) Set of repeatable algorithms indicated as $A_{r}$;

(b) Set of unrepeatable algorithms referred to as $A_{u}$;

A accepting maximal computation allows a TM to perform the same computation again by positioning itself with the head on the first symbol indicated in the table of the transition functions with the $q_{0}$ state. In the same way, the execution of an $A t$ in a system of thermodynamic operations, if it ends with a final state allows a new execution of the same algorithm. So the following Definition can be given:

Definition 39 (Repeatable algorithm). Given a system of thermodynamic operations $S t$ and a thermodynamic algorithm $A t$, if the execution of this algorithm ends with a final state, the $A t$ can be performed again. One indicates this by the symbol $A_{r}$. This occurs if the Ots that belong to it are stationary stochastic matrices with respect to the execution of the same At.

$$
(A t)_{n}:\left\{(A t)_{n \geq 0} \mid\left(c_{0}\right)_{1}=\left(c_{0}\right)_{2}=\cdots=\left(c_{0}\right)_{n},\left(c_{f}\right)_{n} \in F\right\} .
$$

That is, the execution of an $A_{r}$, which ends with a final state, does not significantly modify the components of the matrices of Ots that constitute this At. This is because small variations in the components of the stochastic matrices can lead the algorithm to not be able to finish the computation since the final state could become very unlikely to reach. So one has an $A_{r}$ if there is a symmetry with respect to its execution, if it reaches the final state with the same 
Definition 40 (Not-repeatable algorithm). Given a system of thermodynamic operations $S t$ and a thermodynamic algorithm At, is called not-repeatable, when her computation is finished, if a new accepting computation of it becomes improbable due to changes in the components of the stochastic matrices of its Ots present in the first computation. One indicates this by the symbol $A_{u}$. That is, if these stochastic matrices lose the bonds due to absorbing states.

$$
(A t)_{m}:\left\{(A t)_{m \geq 0} \mid\left(c_{0}\right)_{1}=\left(c_{0}\right)_{2}=\cdots=\left(c_{0}\right)_{m},\left(c_{f}\right)_{m} \notin F\right\} .
$$

Example 13.3. Suppose one has a sheet with a written text, this text can be represented as an At executable by placing a reader on the first letter of the text, with an initial state $q_{0}$. This At represents a computation that ends with the last letter of the text, thus it is also $A_{r}$, as it is executable again at the end of the reading, a second reading and so on. Now it is assumed to execute a second At which has as initial state $q_{0}$ a high energy Ot (e. g. fire), which determines the combustion of the entire sheet. This second $A_{u}$ makes it somewhat unlikely that further execution of both of the previous algorithms will be improbable.

Let us assume to fill a cylinder with a movable wall with an ideal gas at a temperature $T_{1}$. Measurement takes place on the inner walls of the cylinder. The first impact that each gas molecule has against the wall defines the initial state of the $A t,\left\langle q_{0}, s_{1}\right\rangle, \ldots,\left\langle q_{0}, s_{n}\right\rangle$. There will be a finite number of initial symmetry variations and then of Ats. If the system is isolated and the impacts are considered elastic, it will remain indefinitely at the same temperature. However, if the movable cylinder wall is displaced, a second measurement can be made on 
the system. Each collision with the movable wall will result in a terminal pair $\left\langle f_{1}, s_{1}\right\rangle, \ldots,\left\langle f_{m}, s_{m}\right\rangle$ with $f_{m} \in F$ according to Definition (1). The gas collisions with the displaced wall determine a measurable variation corresponding to the termination of a number $m$ of initial Ats. The system temperature drops to $T_{2}$, as the system has done work. Therefore $T_{1}$ must correspond to a number $n$ of $A_{u} \mathrm{~s}$, while $T_{2}$ corresponds to $n-m$ Ats. Then the work done by the gas corresponds to the $m A_{r}$ s. With regard to the efficiency of a thermal machine, please refer to the Section 18.

\section{On the thermal machine and on the PM}

The statements given for a PM in Definition (7) can be applied to a thermal machine. This last machine produces a work from the transfer of energy from a hotter source to a colder source. The two hot and cold sources can be considered as two $S t$, one calls these systems $S t_{h}$ and $S t_{c}$. The thermodynamic entropy of the system must decrease during the transformation. A $S t_{h}$ contains a larger number of $O t$ s because it has a larger number of energy hypersurfaces accessible to symmetry variations than a lower energy $S t_{c}$ system. Furthermore, $S t_{h}$ must have an average information entropy lower than the Ots that compose it. This lower information entropy corresponds to a lower total thermodynamic entropy of $S t_{h}$ compared to $S t_{c}$.

One points out that an increase in energy of a $S t$ corresponds to an increase in Ots accessible to changes in symmetry within $S t$, but this does not determine any change in the value in the components of the matrices that describe the Ots ${ }^{15}$. Thus the matrices associated with the Ots remain invariant during the energy increase. Whereas, if the stochastic matrices associated with the hypersurfaces of energy are no longer stationary, then a variation of the information entropy of these matrices is determined and consequently of the St.

\footnotetext{
${ }^{15}$ One considers an infinitesimal increase in system energy.
} 
A PM represents in part the abstraction of a thermodynamic system. Indeed, the internal energy of any thermodynamic system is represented by heat and work. A PM has a part of Ots with high information entropy and therefore models the effects of energy in the form of heat, and another part of $O t$ with low information entropy that models the effects of the work. While the part not not applicable to thermodynamic systems is represented by the set of logic states $K$ of a PM defined in Eq. (6).

\section{Introduction to Biological part}

In this part the concepts introduced in the mathematical part and in the physical part for the modeling of biological systems will be used. In particular, one shall consider a broader concept, which is defined as a living system. The fundamental thought leading this study is that all living systems try to solve problems. If this statement is admissible and universal for living systems then it is necessary to define which the problem is, in which way one try to solve it and what it means to solve it.

This paper aims to propose an alternative to the understanding of living systems, starting from logical and mathematical bases instead of biochemical or biological ones. If this is correct, it means that the class of living systems may be larger than the one known and based on the DNA molecule. At the end of the discussion two concepts of freedom and happiness will be defined from a mathematical point of view.

The following will be used a terminology not proper of biology. Instead, with a logical mathematical approach to biological systems it is preferred, so that it is easier to demonstrate and refute the different statements. 


\section{On living systems}

A living system can be physically framed as a very complex thermodynamic system. The variety of forms and organizations of these living beings is very wide. It is possible to try to give a definition of a living system based on biochemical structures. This definition is based on the observable fact that all living systems possess DNA and RNA molecules in their structures and are able to reproduce. This definition clearly sets the boundaries of a biological system, but it does not clarify the evolution from non-biological systems to biological systems. Furthermore viruses and viroids have remained on the border of definition between living and non-living systems. Finally, the fact that there are only one type of chiral amino acids in biological systems is not explained.

To address this topic, the definitions given in the mathematical part and in the physical part of this text will be used. Consider a PM in accordance with Definition (7) and let's operate this PM on an alphabet $\Gamma$ formed on energy levels equal to those to which biological systems operate. One considers matter in standard conditions ${ }^{16}$, under which the atomic structures are stable. A symmetry variation on any energy hypersurface at these conditions will represent a $O t$ as defined in Definition (35).

Once the hypersurfaces of energy on which a PM operates are defined, the maximum and minimum limits of the energy of the thermodynamic system are also defined. A biological system operates and is defined within a finite thermodynamic system limited above and below by a maximum and minimum energy levels, and when they are exceeded these structures are not observable or otherwise do not work properly.

The Definition (7) defined a PM without memory if it is not able to memorize the algorithms it creates.

\footnotetext{
${ }^{16} \mathrm{~A} \mathrm{PM}$ is operated in standard chemical-physical conditions but this does not limit the possibility to apply a PM to different conditions and to operate in these different conditions.
} 
A more complex PM is a PM with memory, which is able to memorize the algorithms. So a living system must essentially be formed by two theoretical structures:

Definition 41 (Living system). This set must contain:

(1) PM.

(2) Memory, given by the set of algorithms contained in the system.

Ultimately it is a set of PM with memory, defined in the mathematical part (see Section 5.1).

In biological language, the term memory represents all that is preserved over time in a cell, for example, the cell membrane, its DNA, organelles and all internal structures. At a higher hierarchical scale the memory is represented by the typical structures of multicellular organisms, internal organs, dermis, exoskeleton, nervous system and so on. At a level of even greater complexity, for example for the population, the memory is represented by the individuals who survive, for example the fertile part of the population, the structures that are built and maintained for the following generations as nests, hives, dams in the case of beavers, but also territories occupied and maintained over time, in general ecological niches. One could still generalize by saying that memory represents all that is conserved from one generation to the next, considering also the learned techniques and behaviors. From our peculiar viewpoint, however, it is essential to underline that the memory contains all the biological constructs, ecological niche, behaviors and knowledge that are maintained over time in a specific biological system (organism, population, ecosystem, etc.) whose final goal is not the survival of the biological system in question, but rather to keep the ability to solve problems constant. This viewpoint should not contradict the current theories on the evolution of biological systems as survival is also a problem to be solved and therefore is consistent with the given definition. Moreover, this new definition of the purpose of a biological system allows us to 
explain also observable behaviors that do not seem to have reproduction as the last end.

The stochastic part, fundamental for a PM, can be represented in a biological system as all the biochemical events that take place within the system and which possess an intrinsic uncertainty. For example, mutations of DNA, the motion of molecules and organelles within cells under the effect of heat. In addition in the context of populations, random variations in the position of individuals on a territory are stochastic.

In a living system in general the concept of environment indicates what generally surrounds a living being and what determines its phenotype. Moreover, also the genotype represents, together with the living being, something that remains constant to itself for at least an instant, something observable, therefore it can be seen as a whole as a memory. This memory can be divided into two parts:

(1) External Memory given by the environment, on which the biological system can move remaining intact. This memory in an abstract way can be seen as the tape defined for a TM in Def. (1) as in (Turing 1937).

(2) Internal memory of the living system, this memory represents all that a living system brings with it, of which it retains the symmetry, all that keeps the system equal to itself. The definition set is given in Def. (41).

The system will comply with the energy limits of the thermodynamic system. With regard to a biological system, the energy range within which these structures are observed must be defined. As a comparison, a crystal is of a substance is observed under certain conditions, in such conditions it is stable. In the same way, a biological system can be observed only in conditions where it is stable. A living system represents a more general whole compared to a biological system. Therefore, according to Definition (41), a biological system which is currently 
observed on Earth, can be represented by a set of PMs and by the memorization of a very large number of algorithms.

In conclusion, above considerations, it emerges that the solution of a problem by a living system is possible through two different ways:

(1) If it executes an algorithm already stored within the system itself.

(2) If it builds a new algorithm starting from the structures stored in the system and from the stochastic part by Def. (7) (PM).

The two paths could lead to a huge difference in computational complexity (Hopcroft et al. 2001; Li and Vitányi 2008).

Example 16.1. According to an example taken from Ref. (Lewontin 2002), if one has an electric razor built in the United States it will be suitable to operate with a $110 \mathrm{~V}$ main voltage, while in Europe the main voltage is $220 \mathrm{~V}$, but usually the electric razors have a selector to set the correct voltage value. The razor then contains the instructions to operate at one of the two voltages and it is possible to immediately select one or the other voltage, thus there are then two options or switchable algorithms according to the ambient conditions (110 $V$ or $220 \mathrm{~V}$ ). This corresponds to a solution of a problem of the type presented in the first point above.

Suppose one finds ourselves with an electric razor in another country where the network voltage is $300 \mathrm{~V}$. That electric razor does not provide that voltage and can not be used. In order to use it, one could build a transformer or look for a transformer store. There are many possible solutions, but all of them imply a greater time and complexity than in the previous case, and it could also happen that the shaver can not be used because a suitable transformer is not found. Therefore, using the razor with a $300 \mathrm{~V}$ voltage, corresponds to an application of the second point above. 


\section{${ }_{1235} \quad 17$ Evolution and extinction of a living system}

Any biological system evolves, as it is observed in Nature. In other words, the biological system remains in a probabilistic way equal to itself following an event that instead would tend to vary its symmetry with respect to its configuration. The evolution of a biological system can be represented as an adaptation to a given environmental situation. If such adaptation is possible, the individual or the population will be able to continue to survive. From a more formal point of view the environmental situation can be represented as a PM that produces an $A t$ that can modify the Ats stored in the set formed by an individual or by a population. Being able to adapt to an environmental situation means finding a solution to a problem. In other words, given a living system according to Definition (41) this system will contain a finite number of stored algorithms, and will have to solve a problem if at least one hypersurface of energy outside the living system prevents the termination of an $A t$, contained in the living system . The living system survives, that is, remains the same if, as a result of environmental interference, the memory containing all the algorithms is not damaged. Formally, a living system St consisting of a set of a stochastic subset 
and a subset of memory consisting of algorithms can be represented as:

$$
\mathcal{S}_{v}=\left\{\mathcal{M}_{p}, \mathcal{M}_{1}, \mathcal{M}_{2}, \ldots, \mathcal{M}_{n}\right\}
$$

\footnotetext{
Ats.

${ }^{17}$ The stochastic component of these systems is limited, i. e. it operates on a small set of

${ }^{18}$ Actually one could supposes that it interacts with the whole Universe, but these influences are ignored in this article.
} 
(2) it is not able to formulate new algorithms.

If a living system dies out or dies it no longer has the capacity to find solutions to problems.

Example 17.1. Consider an algorithm for hunting a gazelle by a cheetah. The starting point of this algorithm is represented by the identification of the prey and then the algorithm ends with the killing the prey. This algorithm is reproducible a large number of times, but the cheetah dies if it is no longer able to terminate this algorithm, perhaps because it breaks a leg or maybe because it finds preys with much less frequency.

\subsection{Evolution}

The evolution of a living system allows its adaptation to a thermodynamic system that contains it, which in general is called environment. Evolution is possible only and only if the living system is able to vary, that is to change some characteristics so as to be able to adapt. For example processes such as crossing over or sexual reproduction favor the increase of variability among individuals. Increasing the variability of a living system entails increasing the number of Ats present within that St that is, of the living system considered. In conclusion, based on the observations made so far, it can be stated that the increase in the variability of an $S t$ and in particular of a living system is possible in two ways:

(1) Increasing the number of energy hypersurfaces accessible to the St.

(2) Increasing the entropy of the energy hypersurfaces belonging to the St, without having access to higher energy levels.

As above remarked, any living system operates in a finite range of energy levels, and the number of algorithms it contains are finite in number. Therefore, a living system observed at a given time is able to solve a finite number of prob- 
lems. Increasing the energy of a living system means giving the system new hypersurfaces of energy on which it can build new algorithms. This happens when a living system uses a new source of energy.

Moreover, in order to increase the variability one must ensure that some of the algorithms it contains can be modified, but there is the risk that they can become non-terminal (see Sec. 13). For example, with sexual reproduction the variability of a population increases, but increases the risk that some individuals may fail to procreate, regardless of their adaptability to the environment.

It is therefore deduced that living systems adopt two contrasting strategies to overcome the problems:

(1) While a problem is being addressed by the system, this seeks solutions that minimize uncertainty and therefore tend to minimize information, according to Def. (29), about events that need to happen.

(2) In order to overcome a problem and find a solution, they increase uncertainty and therefore intrinsic variability, this also corresponds to an increase in information on events.

Finally, the consequences of the two systems to increase variability are not the same. If a living system uses higher energy levels to create new variability it must use additional energy sources, then all the algorithms built with these new energy levels will be executable only as long as these levels are accessible. So if it occurs that for some reason the higher energy levels are no longer accessible to the living system, it immediately loses the ability to perform all the Ats that need those energy levels, for example all the biochemical reactions with activation energies higher of the maximum energy available to the living system can not take place.

On the other hand, if a living system increases variability by eliminating constraints (see Sec. 11) on Ots or increasing degrees of freedom for some Ots, it 
is less likely that an external event will block the Ats found as a result of the increase in variability.

Example 17.2. Artificial selection in dairy cattle has led to an increase in the production of cow's milk. This increase in production, in order to be implemented, requires greater energy needs for the selected cows compared to the original population (Clark et al. 2001). Currently there is ample availability of raw materials for the feeding of selected cows, but if this availability drastically decreases the population of selected cows may not survive.

Evolutionary mechanism The fundamental mechanism that makes possible the evolution of a living system consists, on one side, keeping a memory that contains the algorithms for solving outdated problems and on the other side possessing and increasing, when necessary, a stochastic part which allows the living system to look for new problem solving algorithms. Increasing the stochastic part allows to find more algorithms with less complex solutions. One could compare this mechanism to a rope that ends with a loop, and to a blind observer who holds the rope with his hands. The observer represents the memory of the system, the rope the stochastic or variable part. The observer will begin to throw the rope without knowing the environment around, randomly, to look for an anchor point. Many attempts to look for an anchor will fail, if suddenly he finds a foothold, then he will be able to walk to the point found by following the rope. Furthermore, it is assumed that the observer is able to memorize the path he performs. After several attempts he will be able to make paths within that environment. Starting from these paths he will be able to explore more other environments by throwing the rope randomly. If the rope was too long, the observer may not be able to follow the rope to reach a found anchor because there are obstacles that should have been bypassed if the rope had been more short. The stochastic part must be proportional to the size of the memory or the observer. 


\subsection{Final considerations on the evolution of living sys- tems}

By thinking of the fact that a living system can evolve in two ways, some fundamental considerations can be drawn with respect to the main mechanisms for increasing its variability . If one increases the energy available for the living system, it will have a number of hypersurfaces of greater energy available and therefore greater availability of algorithms on which it is possible to construct an evolution. As a simple clarifying example, if an observer placed at the center of a territory to be explored, and he can only walk, the portion of the explored territory will be limited. If, however he will be given more energy through a car, he will be able to explore a larger portion, but if the car becomes unavailable he will soon find himself limited to exploring only the initial territory.

As already seen, it is possible to increase the variability of a living system and therefore the number of available algorithms by increasing the entropy within some $O t$ s, i. e. ensuring that the algorithm that contains it may yield a larger variety of results. For example, if a population of bacteria is treated with $\beta$-lactam antibiotics it is known that at low doses eventually in the bacterial population can be isolated treatment-resistant individuals, which produce enzymes, the $\beta$-lactamases that catalyze the hydrolysis of the ring lactam present in the molecule of $\beta$-lactam antibiotics (Wilke et al. 2005). The production of $\beta$-lactamase does not imply that the bacterial population needs further sources of energy, but instead that it was made possible by modifying some portion of the bacterial DNA.

The second mechanism of increasing the variability of a living system is more durable, secure and sustainable than the first one. The discovery of a new source of energy can instead lead to the extinction of entire living systems if this energy source is no longer available. 
In conclusion, any living system to maintain its symmetry with respect to a variation due to the interaction with the environment implements two mechanisms:

(1) tries to minimize the effects of this variation, ensuring that the variation does not modify its symmetry or its memory to such an extent that it can be extinguished. Therefore the external variation must not irreparably modify the memory of the living system by Def. (42);

(2) increasing the internal variability of your system so as to cancel or reduce the effects of external variation.

Examples of the first case are the shell of a mollusk of the carapace of the turtles which create an impediment to predator attacks or the current families of Elephantidae, which prevent predator attacks only increase the size of their individuals. Or even with polyploidy in many pteridodophytes that should increase resistance to lethal genetic mutations.

The second case occurs when a living system increases the inherent uncertainty or entropy of information, for example by subdividing and distributing the genetic heritage or memory over a greater number of individuals. This happens in the spores of sporophyte plants that produce a large number of spores so that someone is more likely to sporulate.

\section{Freedom and Happiness}

The last topic addressed in this article concerns two concepts born and defined philosophically almost exclusively in the human sphere. It is a natural consequence of previous reflections.

Let $\mathcal{S}$ be a stationary state of any $S t$ and therefore of a living system. A stationary state is a $S t$ without any variation on its hypersurfaces of energy. 
Such that this $S t$ remains symmetrically identical to itself. One indicates with $\operatorname{card}(A)$ the cardinality of a generic set $A$.

Definition 43 (Freedom). Freedom is defined relative to a given $S t_{x}$ and is indicated with $L_{x}$, as the set of all the thermodynamic algorithms $A t_{n}$ that the $S t_{x}$ system is able to execute given the state $\mathcal{S}$ in which it is found.

$$
L_{x}=\bigcup_{n=1}^{k} A t_{n} \quad \text { with } \quad n \in \mathbb{N}^{+} .
$$

Each $A t$ within an $S t$ is distinguishable from another $A t$ from the different initial state.

Proposition 9. Given two systems of thermodynamic operations $S t_{a}$ and $S t_{b}$, with $S t_{b} \subset S t_{a}$ it occurs that:

$$
L_{b} \leq L_{a} .
$$

Proof. The elements of the set $S t_{b}$ are contained within the set $S t_{a}$ therefore the Ats contained in $S t_{b}$ must also belong to the set $S t_{a}$. It follows that according to Definition (43), also $L_{b} \leq L_{a}$.

According to Def. (43) and the previous statement, the following measure of the elements of $L_{x}$ is defined:

Definition 44. Let $\kappa_{1}$ be a positive constant different from zero, and each element of the set $L_{x}$ let be different from the other elements for each pair $\left\langle q_{0}, s_{n}\right\rangle$, then:

$$
\mathcal{L}=\kappa_{1} \cdot \operatorname{card}\left(L_{x}\right) .
$$

From the previous Definition one has that $\mathcal{L}>0$ and in a $S t$ must exist at least an executable $A t$.

Then, given a $S t$, in an initial state $\mathcal{S}$, it is possible that a part of the ther- 
Definition 45 (Happiness). Happiness is defined as the finite set of all $A_{r} \mathrm{~s}$ of the given $S t_{x}$.

$$
F_{x}=\bigcup_{i=1}^{k} A_{r} \quad \text { with } \quad i \in \mathbb{N}^{+} .
$$

From Eq. (43) it follows that:

$$
F_{x} \subseteq L_{x} .
$$

According to the Def. (45) and to the distinguishability of the algorithms from

Definition 47. The efficiency of a $S t$ and therefore also of a living system is given by:

$$
\eta=\iota \frac{\mathcal{F}}{\mathcal{L}} \quad \text { where } \quad \iota=\frac{\kappa_{1}}{\kappa_{2}} .
$$

Proposition 10. Every living system works to maximize the value of $\eta$ or at most to keep it constant. 
Given an Sv according to Definition (41), if any energy hypersurface accessible to this system undergoes a variation due to an $\mathrm{Ot}$, the $\mathrm{Sv}$ reacts so that this variation determines the maximum reduction of information, defined in (29), on the At where the variation is present.

Proof. According to Definition (41) a living system consists of two fundamental parts, a stochastic part with a high entropy and a stable part called memory. Let $S t$ be a thermodynamic system external to a living system but interacting with it by means of an energy hypersurface common to both called $\alpha$. This means that a variation of symmetry that occurs on $\alpha$ involves both the living system and the thermodynamic system. One assumes that to represent the interaction between the two systems there is an At that contains a variation of symmetry on $\alpha$ and let $O t_{\alpha}$ be this variation. One also assumes that the thermodynamic algorithm At belongs to the thermodynamic system external $S t$ because the initial $O t$ is within this system. Without lacking of generality it is also possible to assume that algorithm belong to the living system. If the thermodynamic system external $S t$ interacts on the living system with an $A t$ that one assumes terminal, then it will be possible to measure an effect at the end of the computation. On the side of the living system two situations can occur:

(1) The $O t_{\alpha}$ operation belongs to the stochastic part of the living system or to its PM.

(2) The $O t_{\alpha}$ operation belongs to a thermodynamic algorithm contained in the memory of the living system.

In the first instance, $O t_{\alpha}$ has a low probability of making measurable changes and therefore observable to the living system, as $O t_{\alpha}$ is contained within a set formed by $O t_{s}$ s according to Definition (12.1), then there will exist a considerable number of $A t$ which will contain $O t_{\alpha}$ and in each time the observation will be different. On the contrary, the memory of the living system remains 
unchanged.

In the second instance, instead the variation $O t_{\alpha}$ leads to a modification of an At stored in the living system and therefore to an observable change of this At.

Example 18.2. Consider the following mental experiment. An isolated beaker, filled with water and sealed with a cap. Through the hole steel rod capable of falling over the water can be fitted and using a magnetic switch it is possible to release the rod, which hits the free surface of the water without damaging the beaker. Subsequently the rod is extracted from the water and the system is restored to the initial condition. In this first test, an external observer who observes the system after a sufficiently long time is not able to say whether the rod hit the water or not. In a second test the rod is dropped towards the water with greater speed and this bump causes the beaker that contained the water to break. Subsequently the the rod is put in its initial position. In this case, the observer is able to establish that the rod hit the water.

This experiment shows how a system in which a stochastic part exists can remain unchanged even in the presence of environmental variations.

However, even small variations in the memory of a living system can determine the impossibility of terminating thermodynamic algorithms present inside.

\subsection{Final considerations on the origin of life}

If one assumes molecules such as DNA or RNA as the foundation of life, it is necessary to ask a question about the ordering of the bases in the molecule, for example about what the primordial order should have been. The experiments (Miller and Urey 1959), (Parker et al. 2011) showed how it was possible to create 
organic amino acid molecules from inorganic compounds. But this is not yet sufficient to explain why in the biological systems amino acids are present only in l-form and not in racemic form.

Starting from general theoretical considerations the living systems and therefore the biological ones were defined as a set consisting of two distinct parts, a stochastic part and a deterministic part enclosed in the same set

It could therefore be assumed that in the formation of a living system and in particular of a biological system the following steps would have occurred chronologically passing through these points:

(1) formation of membranes (e. g. phospholipids in biological systems) that by wrapping themselves up isolate a liquid portion and various molecules from the external environment;

(2) selection of metabolic processes within these membranes to withstand environmental energy lowering;

(3) accumulation of memory within these membranes.

Cell duplication should be a process that happened later. This is because also the cellular duplication is a solution to a problem of memory conservation.

\section{Final conclusions}

This paper presents a logical mathematical approach to biological systems allows us to give a description external to them and to construct hypotheses, which that can be verified experimentally. In the mathematical part the concept of symbol of an alphabet has been redefined. The symbol is defined by executing an algorithm that uses another alphabet. It is not the symbol that is postulated, but the algorithm necessary to obtain it. In this way it is possible to apply 
the concept of Turing machine to more complex and real systems if actually, a universal alphabet is not known with which the observable Universe is built. Subsequently, a programming machine has been defined able to formulate a code that is executed by a Turing machine. In its definition, randomness is fundamental. Randomness is expressed at three levels, in the symbol, in the combination of the steps of the code and in the combination of the different algorithms.

The introduction of a randomly based programming machine could open up new scenarios for artificial intelligence studies.

In order to study the living systems, it appeared that first a Section devoted to some physics was needed. Living systems, indeed, can be described as physical systems performing measurements on the real world.

An energy hypersurface is defined as a probabilistic Turing machine represented by a stochastic matrix. The initial state is represented by a stochastic vector. A measurable change, on the phase space, of an energy hypersurface corresponds to a variation of the elements of the stochastic matrix.

The abstract concept of a programming machine is applied to thermodynamics as an abstraction of a thermal machine. Heat and work are rethought, respectively, as stochastic or almost certain symmetry variations of a thermodynamic system. Using this representation through algorithms, conceived in an abstract way as Turing machines, one can deduce that also the measurement of space and time can be represented as algorithms that are reiterated every time a measurement is made. Therefore, the physical world could be represented, in general, by using algorithms, as defined in this paper. This could open a different interpretation of the reality that has not been investigated in this work.

In the biological part the definitions given above in the mathematical and physical parts have been applied. 
The introduction of a measure of the uncertainty of an event (or of the result of an algorithm) is interesting to understand the behavior of biological systems, as they tend to reduce this uncertainty to a minimum. For example, in the human sphere, a measure of information (Shannon 1948) is approximated by the currency of exchange, the latter being an abstract means used as a measure of the uncertainty of trade.

The greater the intrinsic uncertainty of getting a good for a purchaser, the lower will be its monetary value. For instance, no one would buy a ticket for a flight if the probability to arrive to the destination is lower than 20 percent. It might be interesting to look for other forms of approximation of information measurement in other living systems other than humans.

Regarding to the evolution of living systems this work has the fundamental purpose of giving a completely abstract theoretical interpretation, so as to define a more extensive class of systems called living system that should include a subclass represented by biological systems . This should help to better understand how their origin and evolution was possible. Furthermore, the continuity between the non-living world and the living world is underlined.

Obviously, these hypotheses can also be applied in the human sphere. In fact it is seen that living systems that use more energy than others are more vulnerable to extinction, because if some of the highest energy levels are no more accessible, all the algorithms in which they are involved can no longer be executed. For example, the use of high energy levels in industrialized countries would lead to a rapid extinction of their growth models if current energy levels were no longer sustainable. This could be a further and fundamental incentive for the reduction and sustainability of energy use in these countries. As a consequence there is a new argument in favor of reducing carbon dioxide emissions in the atmosphere as well as trying to slow down global warming.

In the animal breeding field, for example, artificial selection leads animals to 
convert food more efficiently and to use more energy to produce more milk and meat. These artificially selected breeds are economically competitive compared to the old domestic animal breeds and they certainly represent an important part of the food sources in the industrialized countries. A drastic decrease in the available energy levels could lead to a rapid decline or even to the extinction of these breeds and this would then produce a food deficit in the industrialized countries. A similar reasoning is possible for the artificially selected plant varieties to adapt to high energy levels.

Finally, structures such as freedom and happiness are defined that deserve further investigation.

Unfortunately it has not been possible to extend the discussion to include a philosophical viewpoint, which would be extremely necessary.

\section{Acknowledgements}

The first ideas of this work were conceived in March 2013.

I thank Prof. Giuseppe Baldovino Suffritti of the University of Sassari, with whom I found the possibility of a very important and profitable discussion, without which this work would have been much more difficult and probably not feasible..

\section{Conflict of interest}

The author declares that he has no conflict of interest. 
1603

\section{Financial support}

This research received no specific grant from any funding agency, commercial or not-for-profit sectors.

\section{References}

Agrios, G. N. (1988), Plant pathology, 5th edn, Elsevier academic press, USA.

Arora, S. and Barak, B. (2009), Computational complexity: a modern approach, Cambridge University Press, Cambridge.

Atkins, P. and De Paula, J. (2010), Atkins Physical Chemistry, 9th edn, Oxford University Press, USA.

Ausiello, G., d'Amore, F. and Gambosi, G. (2003), Linguaggi modelli complessità, F. Angeli, Milano. In Italian.

Ayala, F. J. and Avise, J. C. (2014), Essential readings in evolutionary biology, JHU Press, Baltimora, USA.

Ayala, F. J. and Bendall, D. (1983), Evolution from molecules to man, Cambridge University Press, Cambridge.

Bada, J. L. (1985), 'Amino acid racemization dating of fossil bones', Annual Review of Earth and Planetary Sciences 13(1), 241-268.

Bell, J. S. (1987), Speakable and Unspeakable in Quantum Mechanics, 2th edn, Cambridge University Press, Cambridge.

Chaitin, G. J. (1975), 'A theory of program size formally identical to information theory', Journal of the ACM (JACM) 22(3), 329-340.

Chaitin, G. J. (1990), Information, randomness $\& 3$ incompleteness: papers on algorithmic information theory, Vol. 8, World Scientific Pubblishing, Singapore. 
Chang, Y. T. and Miller, W. H. (1990), 'An empirical valence bond model for constructing global potential energy surfaces for chemical reactions of polyatomic molecular systems', Journal of Physical Chemistry 94(15), 58845888 .

Church, A. (1936), 'An unsolvable problem of elementary number theory', American journal of mathematics 58(2), 345-363.

Clark, J. H. et al. (2001), Nutrient requirements of dairy cattle, 7th edn, National Academy Press, Washington D. C.

Darwin, C. R. (1859), The origin of species by means of natural selection: or, the preservation of favored races in the struggle for life, Murray, London.

Ferraguti, M. and Castellacci, C. (2011), Evoluzione: modelli e processi, Pearson, Italy. In Italian.

Fisher, R. A. (1958), The genetical theory of natural selection, Dover Publications, Inc., New York. Revised and enlarged version of the work originally published in 1930 by Oxford University Press.

Gallager, R. G. (2012), Discrete stochastic processes, Vol. 321, Springer Science \& Business Media, USA.

Gavrilets, S. (2010), 'High-dimensional fitness landscapes and the origins of biodiversity', pp. 45-80. In: Pigliucci and Muller (2010).

Gould, N. E.-S. J. and Eldredge, N. (1972), 'Punctuated equilibria: an alternative to phyletic gradualism?. In: Ayala and Avise (2014).

Gray, R. M. (2011), Entropy and Information Theory, Springer, New York.

Häggström, O. (2002), Finite Markov chains and algorithmic applications, Vol. 52, Cambridge University Press, United Kingdom, Cambridge.

Halliday, D., Resnick, R. and Walker, J. (2011), Fundamentals of physics, 9th edn, John Wiley \& Sons, USA. 
Handey, J. and Live, S. N. (2014), 'Nondeterministic turing machines', https://pdfs.semanticscholar.org/4bd4/ add4e6d06da1fea903e41d0b5d5c55515443.pdf. [Online; accessed 15January-2019].

Hargreaves, W., Mulvihill, S. and Deamer, D. (1977), 'Synthesis of phospholipids and membranes in prebiotic conditions', Nature 266(5597), 78.

Hartley, R. V. (1928), 'Transmission of information', Bell Labs Technical Journal 7(3), 535-563.

Hopcroft, J. E., Motwani, R., Ullman, J. D. et al. (2001), Introduction to Automata Theory, Languages and Computability, Addison-Wesley Longman Publishing Co. Inc., Massachusetts.

Hutter, M. (2007), 'Algorithmic information theory: a brief non-technical guide to the field', arXiv preprint cs/0703024.

Jaynes, E. T. (1957), 'Information theory and statistical mechanics', Physical review 106(4), 620 .

Katz, A. (1967), Principles of statistical mechanics: the information theory approach, WH Freeman, USA.

Kolmogorov, A. N. (1956), Foundations of the Theory of Probability: Second English Edition, Chelsea Publishing Company, USA.

Landauer, R. (1961), 'Irreversibility and heat generation in the computing process', IBM journal of research and development 5(3), 183-191.

Lewontin, R. C. (1983), 'Gene, organism, and environment.. To (Ayala and Bendall 1983) pages 273-285.

Lewontin, R. C. (2002), The Triple Helix: Gene, Organism, and Environment, Harvard University Press, USA. 
Li, M. and Vitányi, P. (2008), An introduction to Kolmogorov complexity and its applications. Texts in Computer Science, Vol. 9, Springer, New York.

Marcus, R. A. (1956), 'On the theory of oxidation-reduction reactions involving electron transfer. i', The Journal of Chemical Physics 24(5), 966-978.

Margulis, L. (1970), Origin of eukaryotic cells: Evidence and research implications for a theory of the origin and evolution of microbial, plant and animal cells on the precambrian Earth, Yale University Press, New Haven, USA.

Margulis, L. et al. (1967), 'On the origin of mitosing cells', Journal of theoretical biology 14(3), 255-274.

Martin-Löf, P. (1966), 'The definition of random sequences', Information and control 9(6), 602-619.

Mencuccini, C. and Silvestrini, S. (1987), Fisica -Meccanica Termodinamica, Liguori editore, Napoli. In Italian.

Miller, S. L. and Urey, H. C. (1959), 'Organic compound synthesis on the primitive earth', Science 130(3370), 245-251.

Motwani, R. and Raghavan, P. (1995), Randomized algorithms, Cambridge University Press, Cambridge.

Parker, E. T., Cleaves, H. J., Dworkin, J. P., Glavin, D. P., Callahan, M., Aubrey, A., Lazcano, A. and Bada, J. L. (2011), 'Primordial synthesis of amines and amino acids in a 1958 Miller $\mathrm{H}_{2}$ S-rich spark discharge experiment', Proceedings of the National Academy of Sciences 108(14), 5526-5531.

Parker, E. T., Cleaves, J. H., Burton, A. S., Glavin, D. P., Dworkin, J. P., Zhou, M., Bada, J. L. and Fernández, F. M. (2014), 'Conducting Miller-Urey experiments', Journal of visualized experiments: JoVE (83), 51039.

Peterson, J. P., Sarthour, R. S., Souza, A. M., Oliveira, I. S., Goold, J., Modi, K., Soares-Pinto, D. O. and Céleri, L. C. (2016), 'Experimental demonstration 
of information to energy conversion in a quantum system at the landauer limit', Proc. R. Soc. A 472(2188), 20150813.

Pigliucci, M. and Muller, G. (2010), Evolution: the extended synthesis, (eds), MIT Press, Cambridge, MA.

Safra, S. (1988), On the complexity of omega-automata, in 'Foundations of Computer Science, 1988, 29th Annual Symposium on Foundations of Computer Science', IEEE, pp. 319-327.

Schnorr, C.-P. (1971), 'A unified approach to the definition of random sequences', Mathematical systems theory 5(3), 246-258.

Shannon, C. E. (1948), 'A mathematical theory of communication', The Bell System Technical Journal 27(27), 379-423, 623-656.

Solomonoff, R. J. (1964), 'A formal theory of inductive inference, part i and part ii', Information and control 7:1-22(2), 224-254.

Solomonoff, R. J. (2011), Algorithmic probability, its discovery, its properties and application to strong AI, in 'Randomness Through Computation: Some Answers, More Questions', World Scientific, Singapore, pp. 149-157.

Tadaki, K. (2008), A statistical mechanical interpretation of algorithmic information theory, in A. Beckmann, C. Dimitracopoulos and B. Löwe, eds, 'Logic and Theory of Algorithms', University of Athens, Association Computability in Europe, Athens, pp. 425,434.

Turing, A. M. (1937), 'On computable numbers, with an application to the entscheidungsproblem', Proceedings of the London Mathematical Society $42,254-546$.

Webster, R. G., Bean, W. J., Gorman, O. T., Chambers, T. M. and Kawaoka, Y. (1992), 'Evolution and ecology of influenza a viruses.', Microbiological reviews 56(1), 152-179. 
Wilke, M. S., Lovering, A. L. and Strynadka, N. C. (2005), ‘ $\beta$-lactam antibiotic resistance: a current structural perspective', Current opinion in microbiology 8(5), 525-533.

Wolfram, S. (2002), A new kind of science, Vol. 5, Wolfram media Champaign, Champaign.

Zenil, H. (2011), Randomness through computation: Some answers, more questions, World Scientific, Singapore.

Zvonkin, A. K. and Levin, L. A. (1970), 'The complexity of finite objects and the development of the concepts of information and randomness by means of the theory of algorithms', Russian Mathematical Surveys 25(6), 83-124. 